\title{
Chapter 4
}

\section{Carbon Nanotubes-Polydimethylsiloxane Sensor}

\begin{abstract}
This chapter depicts the design, fabrication, and employment of the first novel sensor prototype formed from Carboxylic acid functionalized Multi-Walled Carbon Nanotubes (MWCNTs) and Polydimethylsiloxane (PDMS). Casting and laser cutting techniques were used to develop the patches where the electrodes were curved out off a nanocomposite layer that was formed by mixing MWCNTs and PDMS. The sensors were then employed for monitoring limb movements and respiration by attaching them to the joints of the limbs and lower part of the diaphragm. They were also deployed for low-pressure tactile sensing purposes.
\end{abstract}

\subsection{Introduction}

The design, fabrication, and execution of novel flexible sensor prototypes were done using PDMS and MWCNTs are elucidated in this chapter. The utilization of polydimethylsiloxane (PDMS) was done as the substrate and a nanocomposite formed from PDMS and Carbon Nanotubes (CNTs) as electrodes. PDMS is one of the materials that has been widely used (Armani et al. 1999; Fujii 2002; Jo et al. 2000) for fabricating flexible sensors due to specific advantages like low cost, non-toxicity, inertness and hydrophobic nature. CNTs were preferred as the conducting material over other nanoparticles due to certain advantages like their biocompatibility, high flexibility, high resistance towards concerning the changes in temperature, low stiffness, and high tensile strength. Carboxylic group (-COOH) functionalized multi-walled carbon nanotubes (MWCNTs) were considered for developing these sensor patches due to their enhanced dispersing capability within a polymer in comparison to non-functionalised or single-walled carbon nanotubes (SWCNTs). An enhanced interfacial bonding is formed between the 
nanotubes and the polymer, thus resulting in increased electrical conductivity of the resultant nanocomposites. Interdigital electrodes were patterned on the conductive layer to form sensors for noninvasive and single-sided measurements. The interdigitated patterning was done using laser ablation (Gower 2000; Snakenborg et al. 2004) using a $\mathrm{CO}_{2}$ laser cutter.

In comparison to other fabrication techniques like 3D printing (Lam et al. 2002), photolithography (Herzer et al. 2010) or inkjet printing (De Gans et al. 2004), laser cutting has certain distinct advantages like ease of sample preparation without the necessity of the requirement of any template to develop the electrodes. It also forms very thin and flexible final products while forming smooth edges that are perpendicular to the surface. The employment of these formed sensors was done by attaching them to the skin to measure physiological movements and respiration of different people. They were also employed for tactile sensing by exerting low pressure on the sensing area of the patches via attaching them on the finger.

\subsection{Fabrication of the Sensor Patches}

Figure 4.1 shows the schematic diagram of the fabrication process. PDMS (SYLGARD ${ }^{\circledR}$ 184, Silicon Elastomer Base) was formed at a ratio of 10:1 for the base elastomer (pre-polymer) and curing agent (cross-linker) and cast on a Poly (methyl methacrylate) (PMMA) template. PMMA was used as the main template for casting the PDMS due to its impassiveness towards PDMS, and the ease of peeling off PDMS from the template without the requirement of any additional step. A casting knife (SHEEN, 1117/1000 mm) was used to adjust the thickness of the PDMS cast on the PMMA to $1 \mathrm{~mm}$. Then the desiccation of the sample was done for 2 hours to remove the air bubbles trapped in the sample. Then the curing of the sample was done $80{ }^{\circ} \mathrm{C}$ for 8 hours to solidify the substrate of the sensor patch. This was followed by forming a nanocomposite layer on top of the cured PDMS by mixing functionalized MWCNTs (Aldrich, 773840100G) and PDMS. 


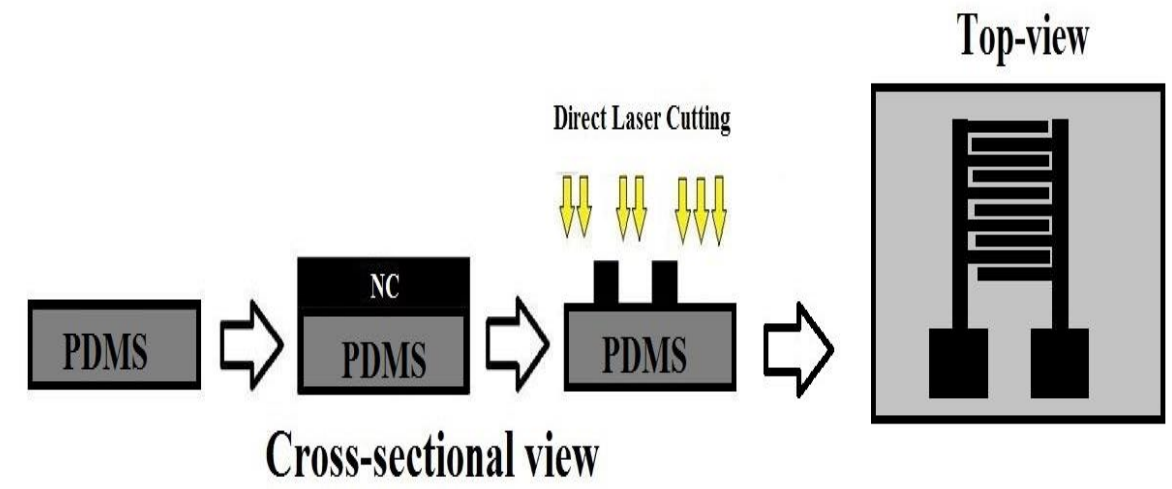

Figure 0.1 Schematic diagram of the fabrication steps. PDMS: Polydimethylsiloxane. NC: Nanocomposite.

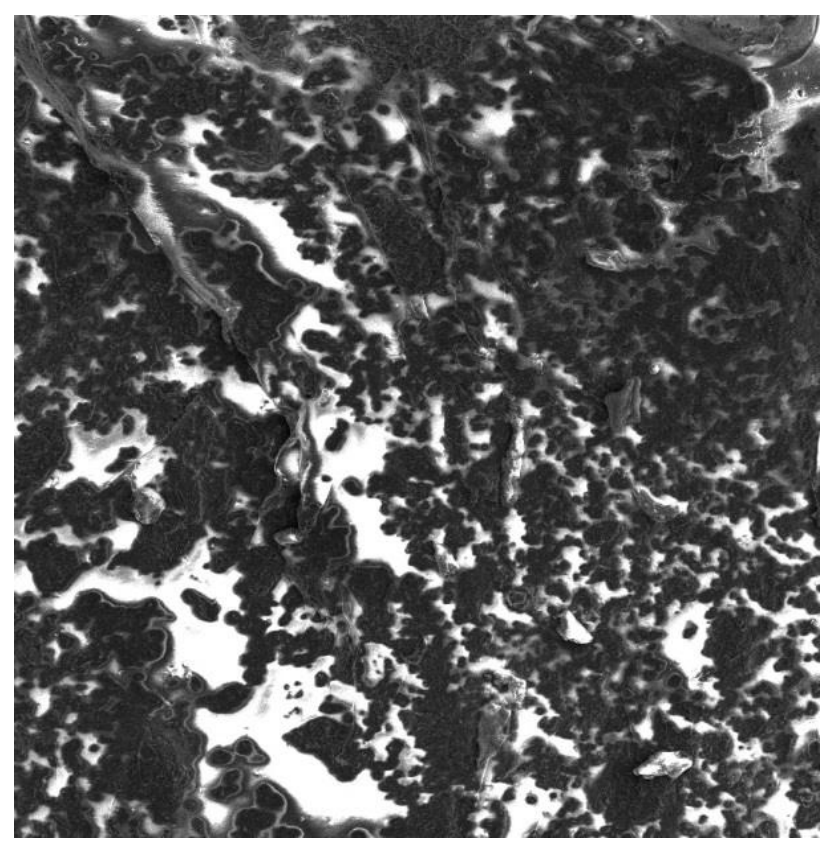

Figure 0.2 SEM top-view image of the nanocomposite consisting of CNTs (4\% wt.) and PDMS.

4 wt. \% of CNTs was considered as the optimal value by obtaining a trade-off between the electrical conductivity and mechanical flexibility of resultant nanocomposite. An SEM image of the 
developed nanocomposite is shown in Figure 4.2. The white and black regions in the image represent the mixed PDMS and MWCNTs respectively. Similar to the cured PDMS, the height of the nanocomposite layer was adjusted using the casting knife to around 600 microns, which was then subsequently desiccated for 2 hours and cured at $80{ }^{\circ} \mathrm{C}$ for 8 hours. Laser cutting (Universal Laser Systems) was then conducted on the cured nanocomposite layer to form the defined electrode patterns. Figures 4.3 shows the individual steps of fabrication that were followed to form the sensor patches. The electrodes of the formed patches were tested with a series of shortcircuit connection tests to decide the combination of the power and speed parameters of the laser system. A combination of $24 \mathrm{~W}$ and 70 $\mathrm{m} / \mathrm{min}$ was eventually turned out to be comparatively the most viable one that was set for forming rest of the sensor patches.

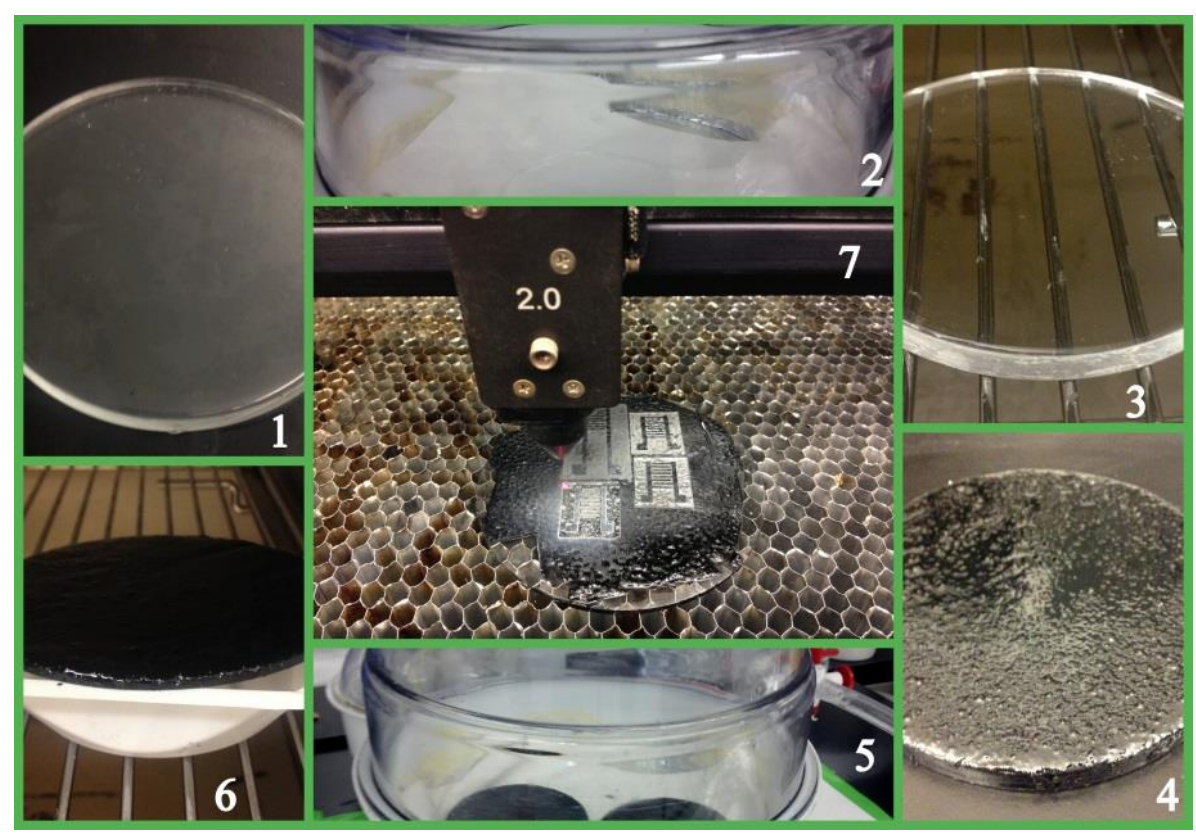

Figure 0.3 Fabrication steps: (1) Casting of PDMS, (2) Desiccation of PDMS, (3) Curing of PDMS, (4) Casting of nanocomposite (NC), (5) Desiccation of NC, (6) Curing of NC, (7) Laser patterning of electrodes. 


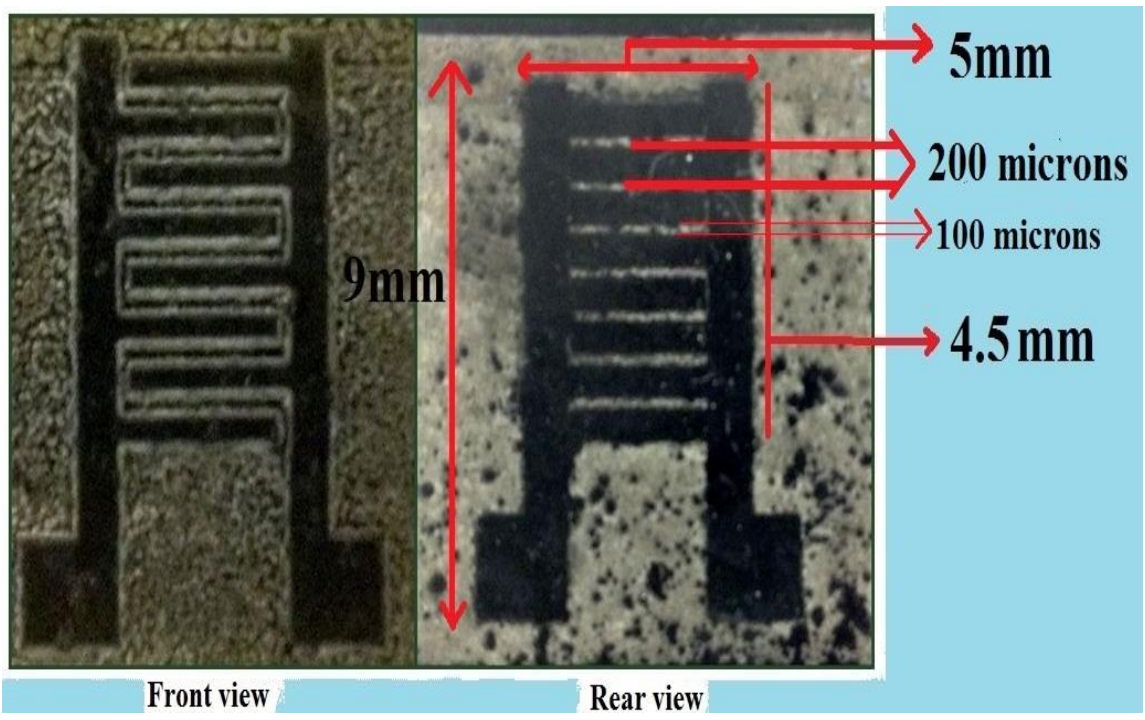

Figure 0.4 Front and rear-view images of the sensor patch.

Table 0.1 Power and speed combinations of the laser cutting tool to obtain different electrode thicknesses.

\begin{tabular}{|c|c|c|c|}
\hline Power $(\mathbf{W})$ & $\begin{array}{c}\text { Speed } \\
(\mathbf{m} / \mathbf{m i n})\end{array}$ & $\begin{array}{c}\text { Z-axis } \\
(\mathbf{m m})\end{array}$ & $\begin{array}{c}\text { Thickness of the } \\
\text { electrodes } \\
\text { (microns) }\end{array}$ \\
\hline $\mathbf{1 . 2}$ & 4.2 & 2 & 137 \\
\hline $\mathbf{2 . 4}$ & 7 & 2 & 140 \\
\hline $\mathbf{1 2}$ & 42 & 2 & 150 \\
\hline $\mathbf{2 4}$ & 70 & 2 & 175 \\
\hline $\mathbf{4 2}$ & 126 & 2 & 180 \\
\hline
\end{tabular}

Figure 4.4 shows the front and rear views of the developed sensor patches. The black spots present in the two views of the sensor patches are unscanned agglomerations of CNTs that lie within the PDMS layer. Table 4.1 shows a combination of power and speed settings that were tested to achieve the optimal combinations for the sensor 
patches. Power (W) defined the intensity of how energetically the laser fired on the material. Speed $(\mathrm{m} / \mathrm{min})$ defined the rate of movement of the laser nozzle over the substrate in the $\mathrm{X}-\mathrm{Y}$ directions. $\mathrm{Z}$-axis was altered to determine the focal point of the laser beam on the substrate material. A profilometer (XP-200) was used to determine the thicknesses of the formed electrodes. When the sensors were developed with very low power and speed settings, a hardening effect of the patches was observed which caused the nanocomposite layer to come off upon application of stress on the patches.

\subsection{Frequency Response and Stress-Strain Measurements}

Impedance measurements of the sensor patches were taken using a Precision Impedance Analyser (Agilent 4294A). Open and short circuit calibrations were done before the measurements to minimize the effects of stray capacitances. A frequency sweep was done between $10 \mathrm{kHz}$ and $10 \mathrm{MHz}$ to obtain the corresponding impedances $(\mathrm{Z})$ and phase angles $(\Theta)$ values as depicted in Figures 4.5 and 4.6 respectively.

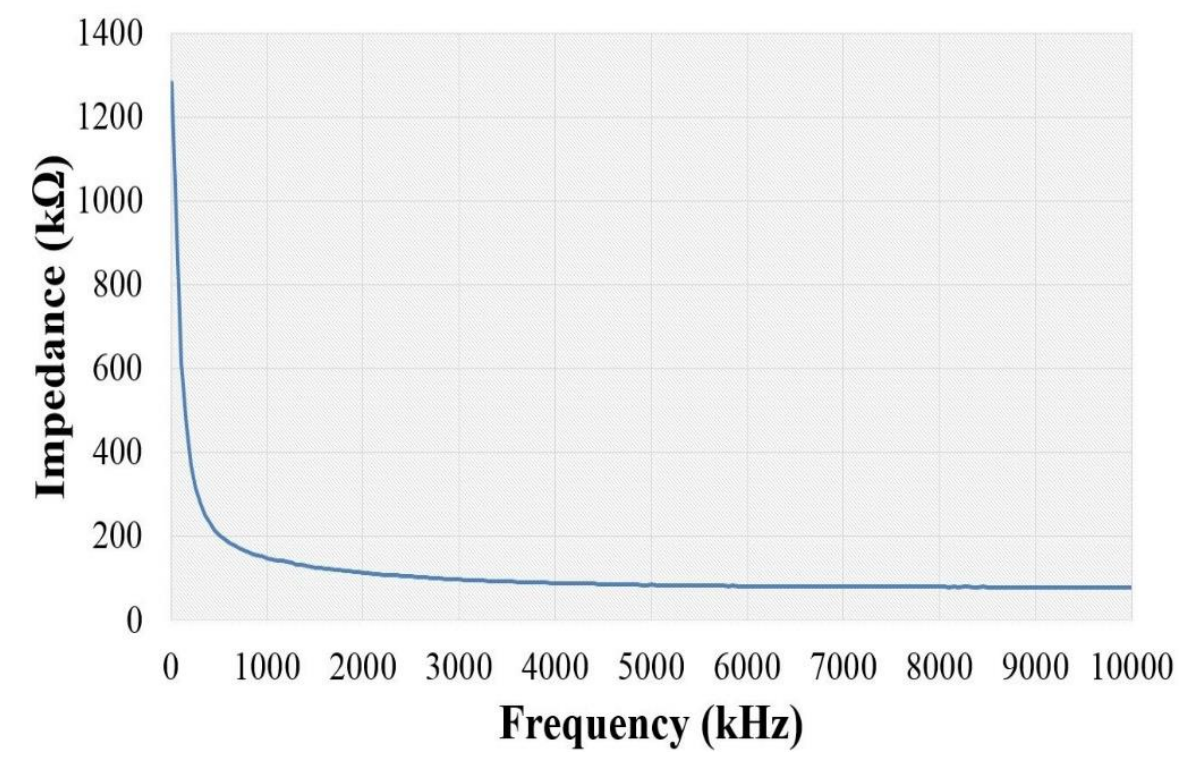

Figure 0.5 Impedance behavior of the sensor patch as a function of frequency. 


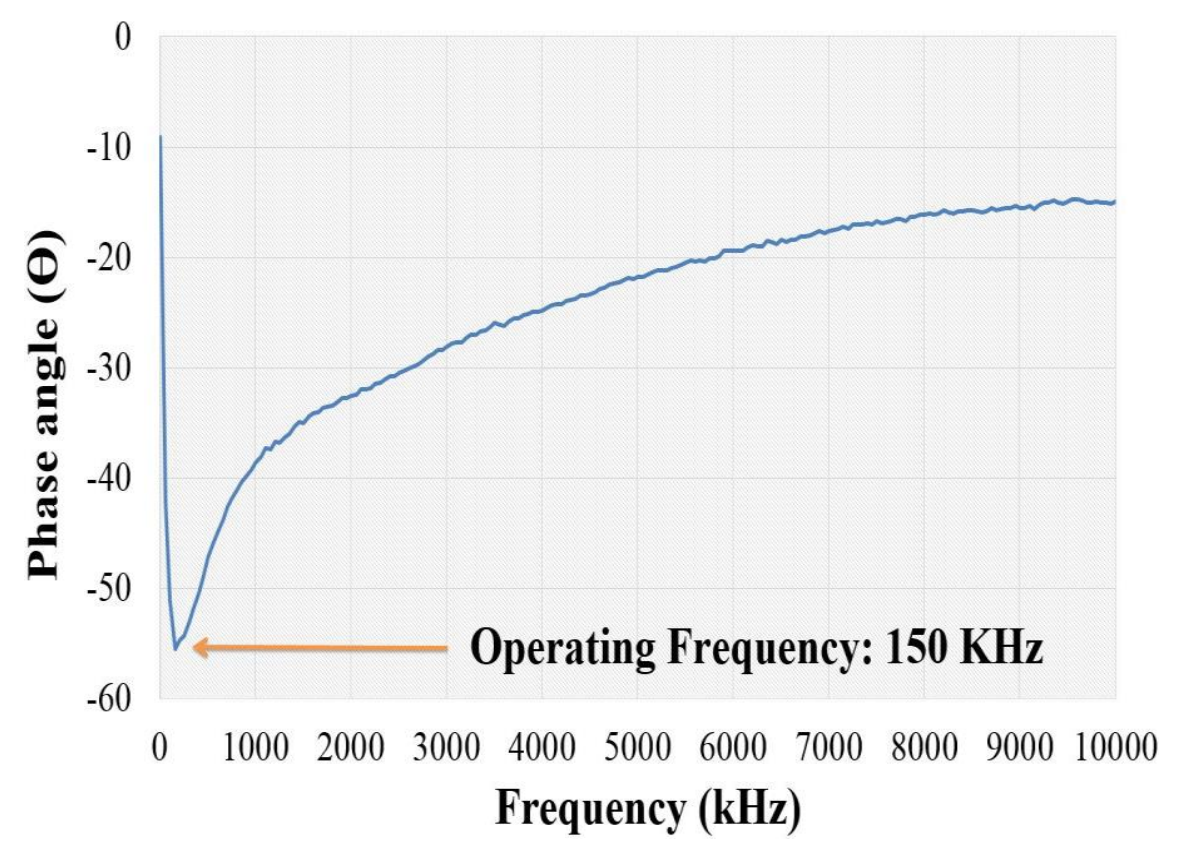

Figure 0.6 Phase angle of the sensor patch as a function of frequency.

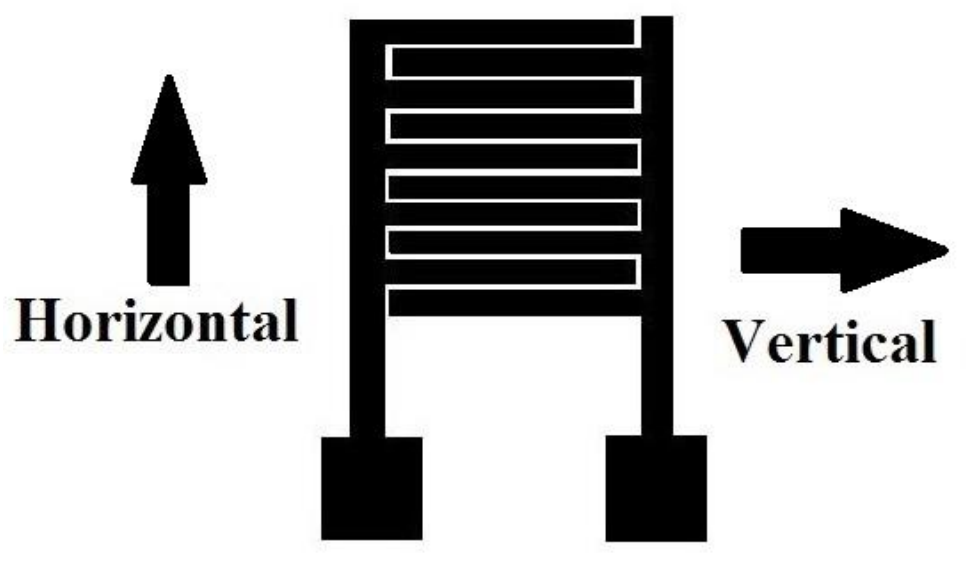

Figure 0.7 Directions of the applied stresses for the stress/strain characterization of the sensor patch. 
The capacitive nature of the sensor patches caused to have a peak of the phase angle at a frequency value of $150 \mathrm{kHz}$. This frequency of $150 \mathrm{kHz}$ was fixed to perform further characterization and experiments. The stress-strain experiments of the sensor patches were performed using an INSTRON extensometer tensile/compressive force testing system (VS02477052 R: F). Figure 4.7 shows the directions of the stresses that were being applied on the sensor patches. It is seen from Figure 4.8 that an anticipated stress-strain relationship (Frankland et al. 2003) was obtained after the stress was applied in the horizontal direction but not in the vertical direction. This was due to the anisotropic geometry of the electrode design. The fracture points acquired from the tensile stress were $(1420 \mu \mathrm{m}, 2060$ $\mathrm{mN})$ and $(-1680 \mu \mathrm{m},-840 \mathrm{mN})$.

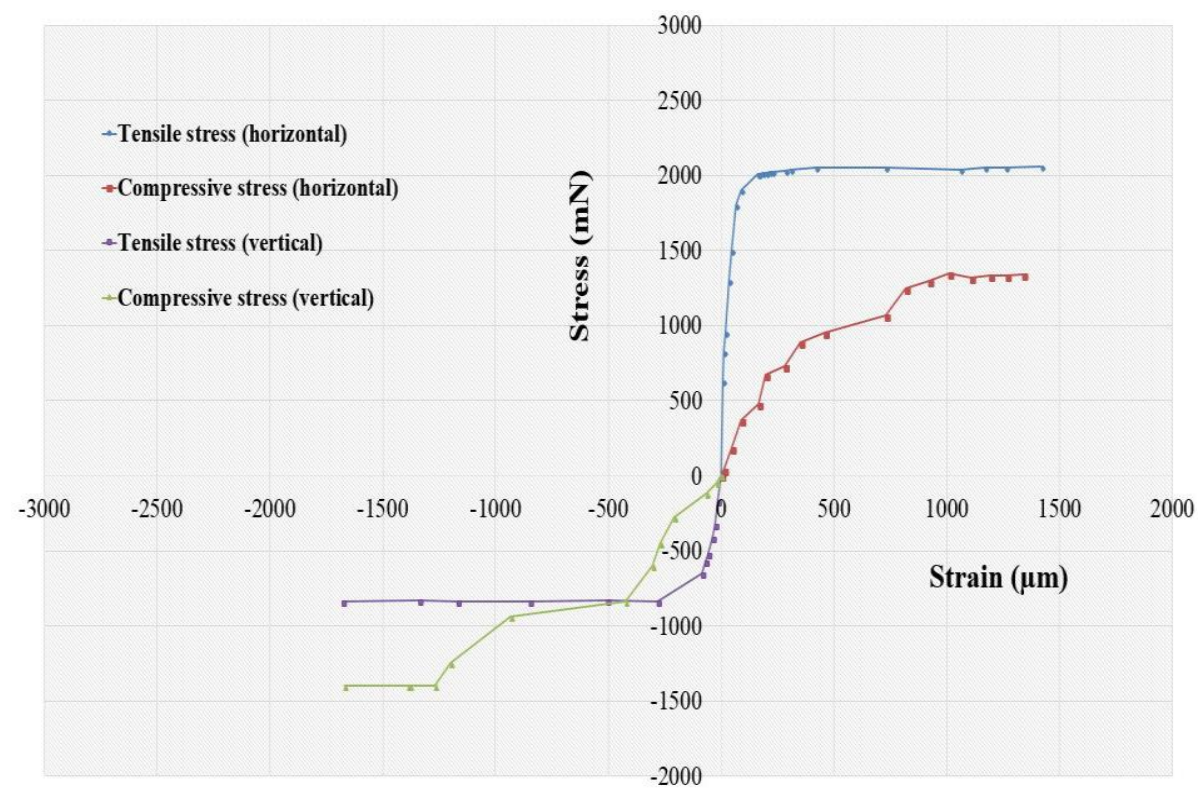

Figure 0.8 Stress-strain relationship of the sensor patch.

The lower limit of the negative strain was a result of the excessive bending of the sensor patches. The relationship between capacitance and strain of the sensor patches was obtained by exerting strain at three different frequencies to obtain the respective capacitances. It is seen from Figure 4.9 that there was a prominent change in capacitance 
values concerning applied strain, the highest was being obtained at the operating frequency $(150 \mathrm{kHz})$. The sensitivity was calculated from the curve at the optimum frequency of $150 \mathrm{kHz}$ from the given formula,

Sensitivity $=\frac{\Delta C}{\Delta \text { Strain }}=\frac{179.2-122.3}{1187.5-728}=0.124 p F / \mu m$.

The linear portion of the frequency line was obtained between $610 \mu \mathrm{m}$ and $1120 \mu \mathrm{m}$. The saturation value at $150 \mathrm{kHz}$ was obtained at 2160 $\mu \mathrm{m}$.

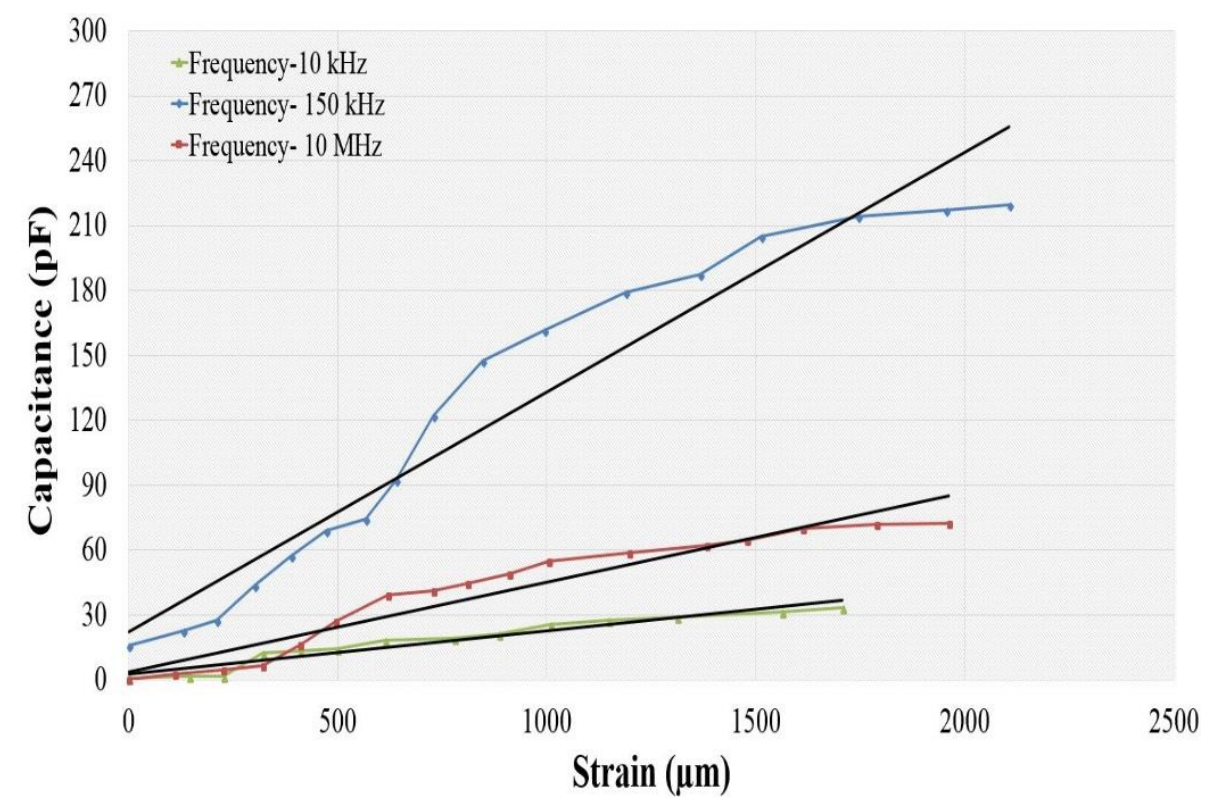

Figure 0.9 Relation between capacitance and strain of the sensor patch in the horizontal direction.

\subsection{Monitoring of Physiological Parameters}

The first group of experiments performed with the CNT-PDMS sensor patches was to determine some of the physiological parameters of human beings. The sensor patches were attached to specific locations of the human body to determine the movement of limbs and 
respiration. The idea behind choosing these two applications is to highlight the applications of CNT-PDMS sensor patches in the biomedical sector. Due to the distinct advantage of mechanical flexibility of these sensor patches, they would certainly be useful to detect the voluntary physical changes taking place on a person's body. Some of the potential applications lie in their deployment to determine the post-rehabilitative movements of the patients who just suffered from a stroke or muscle spasms. The concept of the use of sensors to monitor people's health and lifestyle has been capitalized for the past two decades (Altun and Barshan 2010; Najafi et al. 2003) where different types of sensors have been deployed to monitor certain activities and physiological parameters of individuals to analyze and generate a pattern of the human behavior (Kelly et al. 2013; Malhi et al. 2012; Suryadevara and Mukhopadhyay 2012). Flexible sensors are one area where prominent research work (Arena et al. 2010; Ashruf 2002; Briand et al. 2011; Charton et al. 2006) has been largely done in recent times. Lightweight, low cost of fabrication and high longevity are some of the ground reasons for their increased preferences over rigid substrates. The sensors fabricated for smart home usage are mainly used for specific monitoring purposes like PIR sensors (Hayes et al. 2009), pressure sensors and other ones.

Multiple parameter monitoring is of great interest as a result of the disadvantage caused by a large number of sensors used for individual applications. This is due to the reduced cost of a single sensor that can be used for multiple functionalities. The fabrication of the sensor patches explained here is much simpler regarding the quick and cheaper fabrication process in comparison to previously developed sensors, which were developed for multiple functions containing a coil (Kaniusas et al. 2006; Mehnen et al. 2004; Pfützner et al. 2006) operating on a magnetic principle.

Noteworthy research work has also been done on the determination of joint and limb movements. But the majority of them is related to fixed sensors (Crabtree et al. 2003) or the study involving an artificial robot (Aminian and Najafi 2004) to determine the human behavior. Wearable sensors (Nguyen et al. 2011), accelerometers (Yang and Hsu 2010) are other similar sensors have been used to monitor human movement. Shoe sensors (Sazonov et al. 2011) and braces (Bell et al. 2015) are some of the types of wearable sensors that 
were used for monitoring of physical activities that involves limb movements. The existing sensors have certain disadvantages like constant wearing for ubiquitous measurements, complicated circuitry of the sensing system which require specific expertise to operate them. So, it is state-of-the-art to develop a simple, non-invasive, sensing device that can monitor the movements when they are attached to certain regions. Research work to monitor the rate of respiration has also been done earlier using rigid and flexible sensors. The photoplethysmographic technique (Leonard et al. 2006; Nilsson et al. 2000) is widely preferred for the detection of the rate of respiratory. But due to the complexity, time consumption and requirement of technicians of this technique, researchers have worked on piezoresistive (Reinvuo et al. 2006), fabric-based (Jung et al. 2006; Merritt et al. 2009) and optical sensors (Warkentin et al. 2007) as an alternative to monitor the rate of respiratory. The technical complexity, cost and specific location of the subject during the experimental procedure are some of the demerits of these techniques. Monitoring of respiration and other physiological parameters has also been considered using types of sensors like PVDF-based piezoelectric sensors (Bu et al. 2007; Chiu et al. 2013). But the demits of using PDVF-based sensors are their strong dependency on temperature and high hysteresis. Apart from this, there are different types of force sensors available in the market that are used for these applications. Table 4.2 classifies some of them based on price, size and certain applications related to the monitoring of physiological parameters. It is seen that either the price of the sensors is very high, or the size of the sensor size is reasonably large. In the experiments shown in the subsequent section, we showed the change in the capacitance values of the developed sensor patches by simply attaching them to the joints of the limbs and lower part of the diaphragm of an individual. The inhalation and exhalation rates for the rate of respiration were monitored based on the induced strain on the sensor patch. This idea is useful for certain applications like the anomaly caused in the rate of respiration as a result of hypoxemia and hyperemia, which can be subsequently analyzed by determining the differences between the capacitance values between a healthy person and a patient. 
Table 0.2 Comparison of different force-sensing resistors available in the market.

\begin{tabular}{|l|l|l|l|l|}
\hline Sensor & $\begin{array}{l}\text { Size } \\
(\mathrm{mm})\end{array}$ & $\begin{array}{l}\text { Force } \\
\text { sensing } \\
\text { capacity } \\
\text { (lbs) }\end{array}$ & $\begin{array}{l}\text { Price } \\
\text { (USD) }\end{array}$ & Application \\
\hline $\begin{array}{l}\text { RB-Phi- } \\
\mathbf{1 2 1}\end{array}$ & $25^{*} 11$ & 11.24 & 45.00 & $\begin{array}{l}\text { Pressure-sensitive touch } \\
\text { user interface. }\end{array}$ \\
\hline $\begin{array}{l}\text { Flexiforce } \\
\text { A101 } \\
\text { Sensor }\end{array}$ & $15.6 * 7.6$ & 10 & 34.00 & $\begin{array}{l}\text { Bed monitoring systems, } \\
\text { force-sensitive video } \\
\text { games. }\end{array}$ \\
\hline $\begin{array}{l}\text { SKU: SEN } \\
\text { 09376 }\end{array}$ & $3.5^{*} 3.5$ & 4.49 & 14.28 & $\begin{array}{l}\text { Tactile sensor for robotic } \\
\text { appendages. }\end{array}$ \\
\hline SEN-09375 & $2.375^{*}$ & 2.24 & 6.75 & $\begin{array}{l}\text { Bicycle handlebar glove, } \\
\text { human symbiotic robot. }\end{array}$ \\
\hline
\end{tabular}

\subsubsection{Experimental Setup}

To verify the functionality of the sensor for biophysical parameter monitoring, the patch was fixed to the skin using biocompatible tapes (VHB 3M RP) as shown in Figure 4.10. The sensor was fixed only after the skin was completely dried to reduce the effect of sweat or water due to the attachment of the tapes. The existence of sweat on the skin would lead to an additional capacitive layer between the sensor and skin, causing erroneous results. 


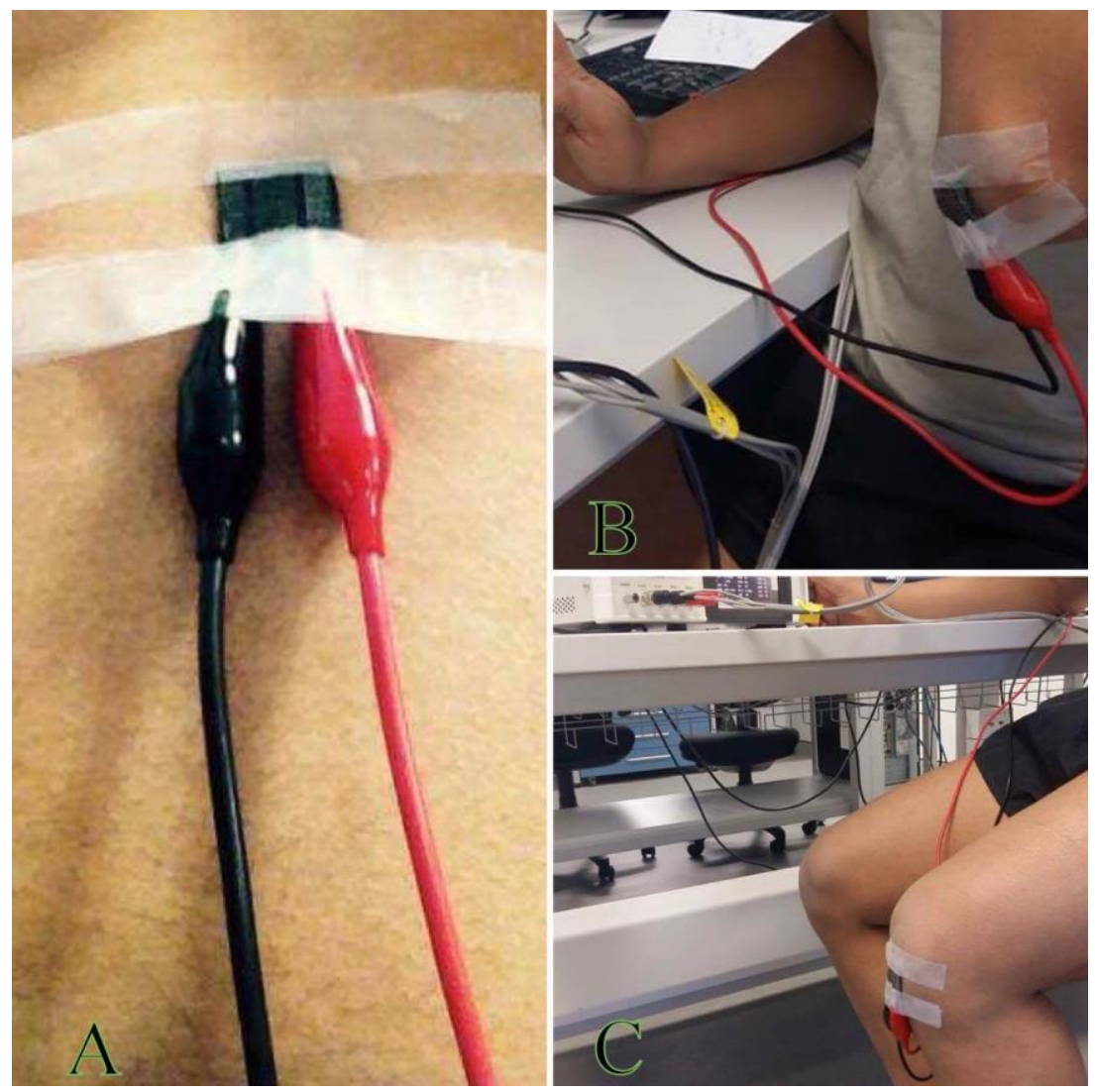

Figure 0.10 Sensor attachment for the biophysical parameters: (A): Monitoring of respiration with the sensor attached on the lower part of the diaphragm, (B): Sensor attached on the elbow to monitor arm movement, (C): Sensor attached on the knee to monitor leg movement.

The experimental results regarding the change in capacitance of the sensor were taken by a Precision LCR meter (E4980A) at 150 $\mathrm{kHz}$. BNC-to-alligator clips were connected to the LCR meter from one end and the sensor patch from the other which was attached to the body. Respiratory measurements were taken by attaching the sensor patches at the lower end of the diaphragm of a person. The readings were taken at two different conditions. The connection of the sensor patches was made at the trochlea of the elbow and the patella of the knee to detect the limb movement. The terms 'flexed' and 'extended' 
mentioned in the figures refer to states of the limb positions. The movement of the arms was done from a fully extended position, i.e., resting on the table to a fully flexed position via bending the elbow at different angles. The movement of the leg was done similarly by causing an of bending of the knee from an extended to a flexed position. The position of the sensor on the body is crucial regarding the life span and reproducibility of the results. For example, if the sensor is positioned in a tilted position, the stress exerted on the sensor patch would not be distributed equally. This would lead to a nonuniform stretching of the patch, thus generating an unequal interdigital distance $(d)$ between the electrodes and as a result, producing erroneous results.

\subsubsection{Results and Discussion}

People of different age groups were considered for testing for monitoring of movement of limbs and respiration to affirm the functionality of the sensor patch. Figures 4.11 - 4.14 show the response of the sensor when the subject was at rest and an oscillatory movement was done for the limbs. The results show that the detection of the movement of the limbs can be clearly done by the connected sensor patch. The limbs were flexed up to an angle of $130^{\circ}$, considering a reference of zero degrees for the extended limb. Regarding the results, there are a few issues that can be addressed to optimize the performance of these sensors for this application. For example, in Figures 4.11 and 4.12, minute fluctuations are observed during the flexed state. This can lead to contradictory assumptions of the condition of the limb. One of the reasons for this could be a movement of the cables during the movement of the limbs. This issue could be rectified by the wireless operation. Another reason could be the loosening of the sensor patch during the movements of the limbs which lead to the observed artifacts. The signals in the flexed position showed some extent of contradictory values. The reason for this could be that the movement of the limbs was not completely identical for two different cycles. Figures 4.15 and 4.16 show the sensor response when the person is at motion. The changes in capacitance values was monitored when the person moved his limbs while walking. 


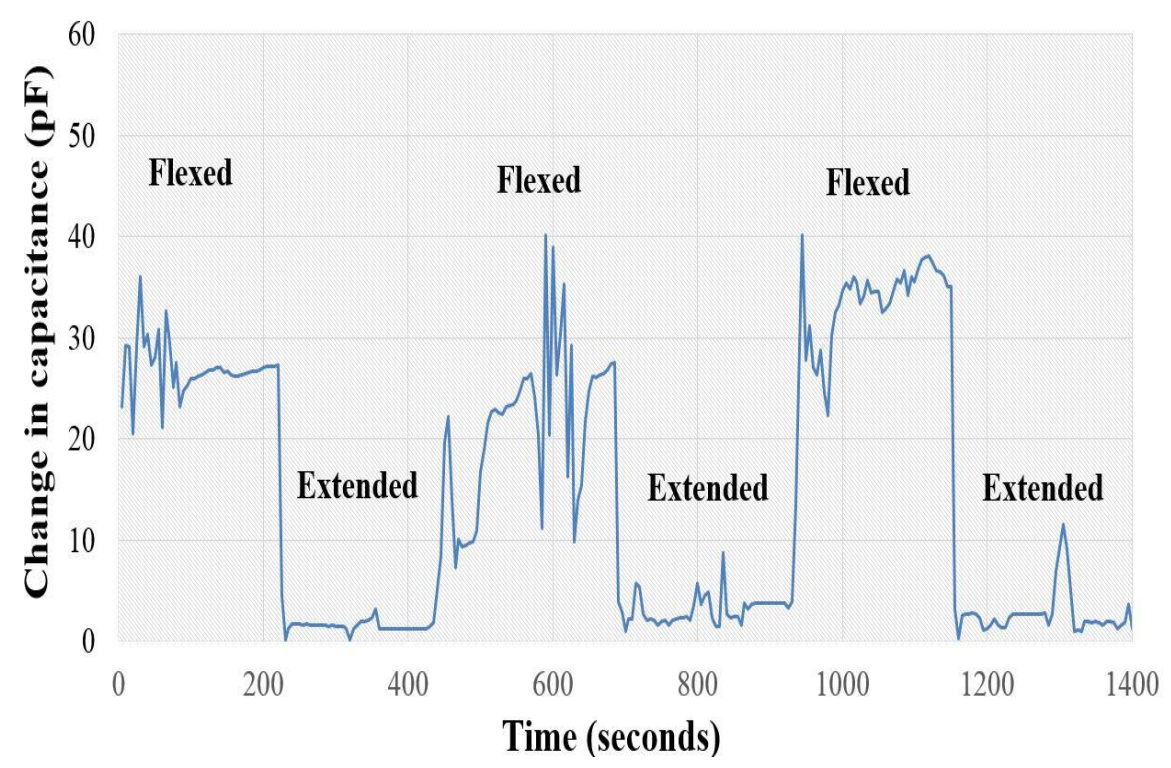

Figure 0.11 Detection of left-arm movement when the subject is at rest.

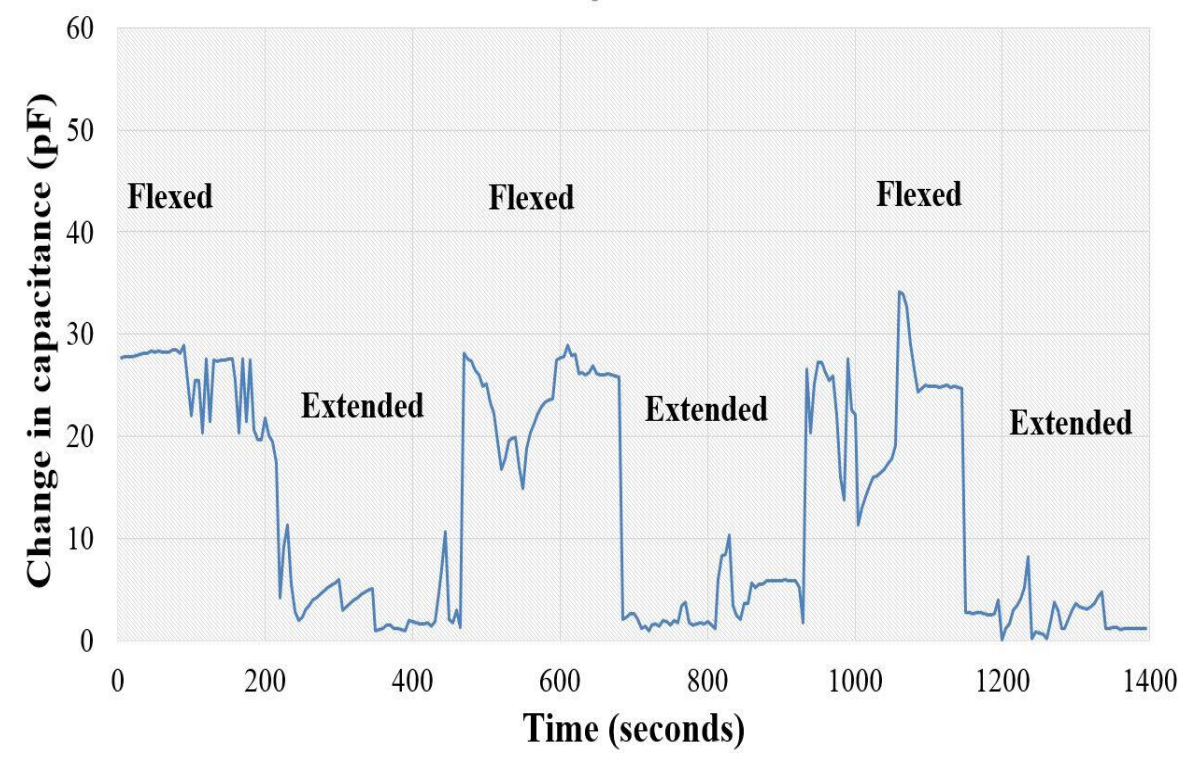

Figure 0.12 Detection of right-arm movement when the subject is at rest. 


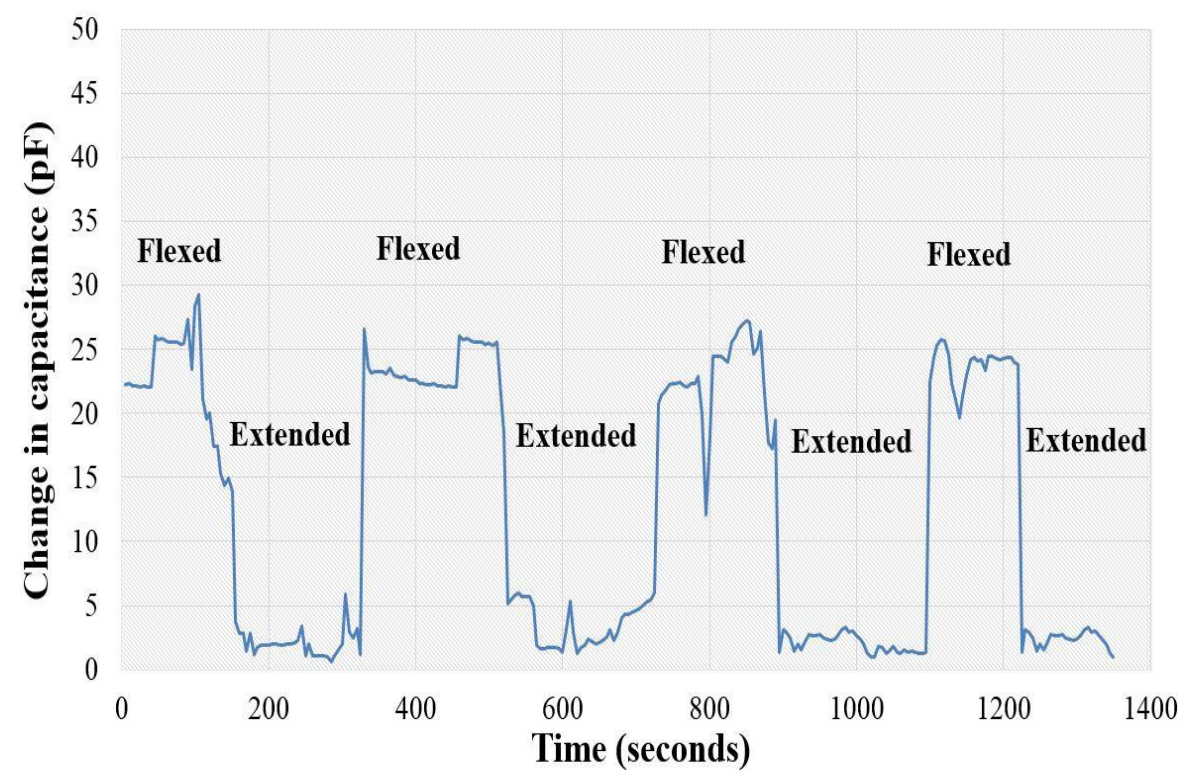

Figure 0.13 Detection of left-leg movement when the subject is at rest.

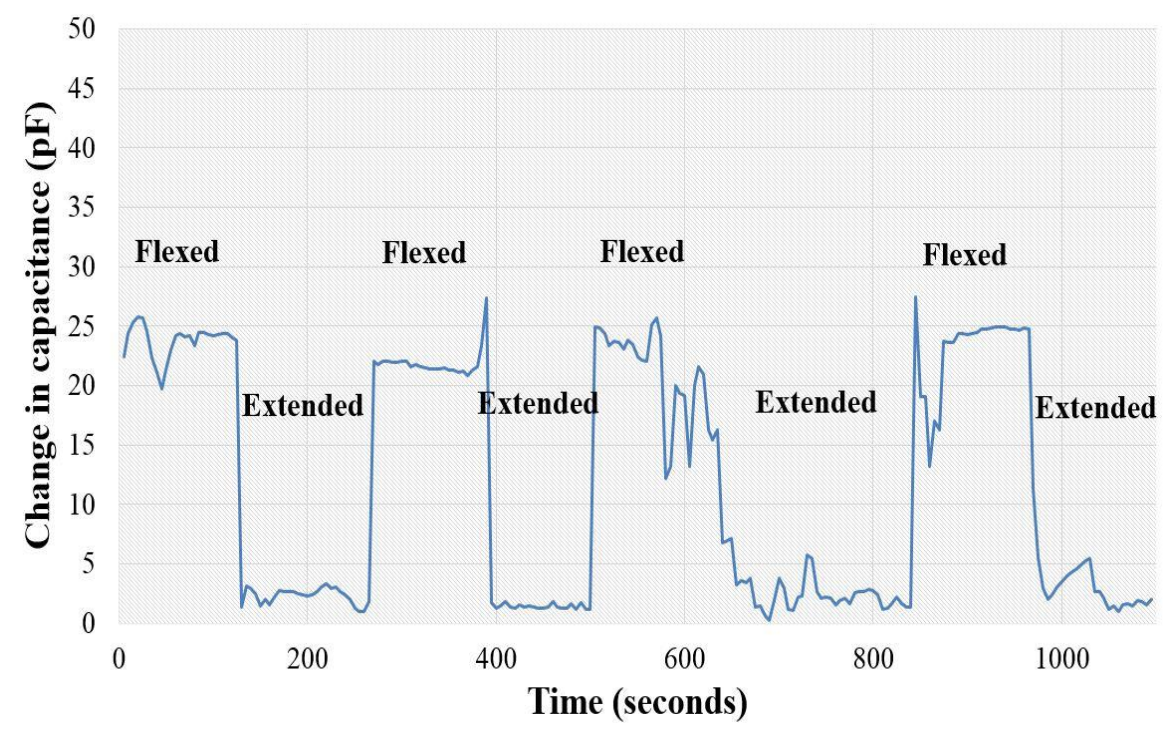

Figure 0.14 Detection of right-leg movement when the subject is at rest. 


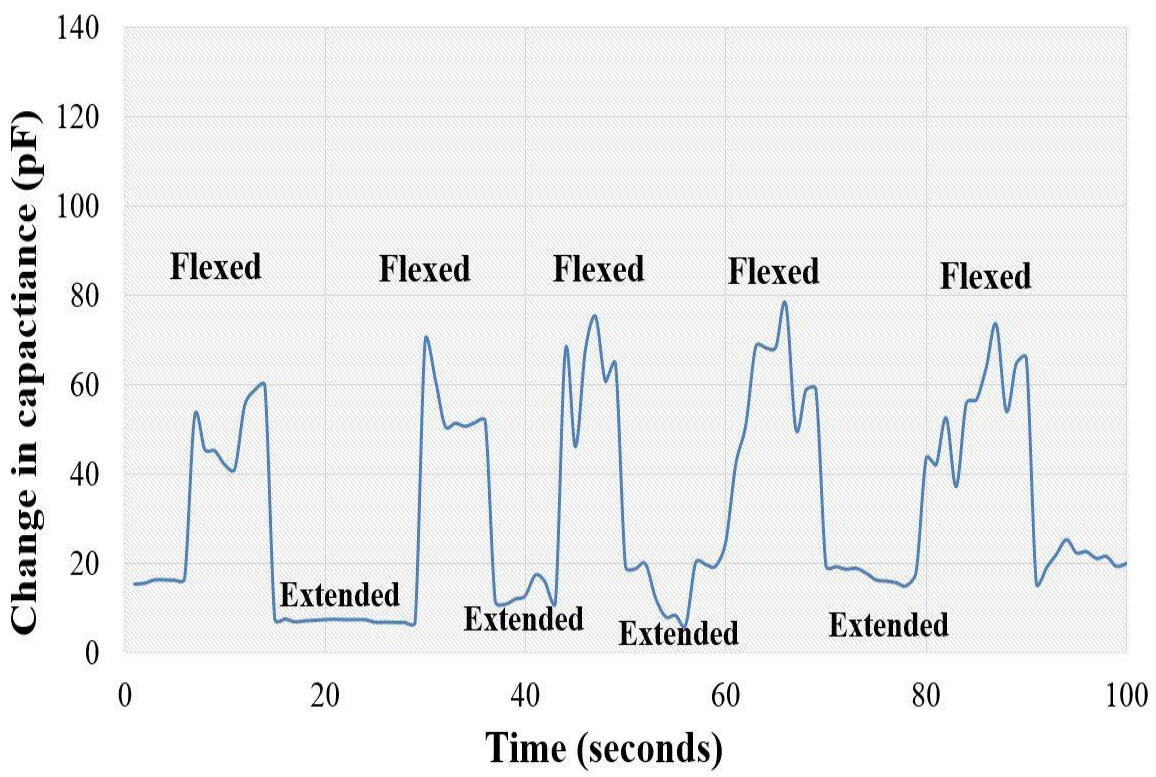

Figure 0.15 Detection of right-arm movement when the subject is in motion.

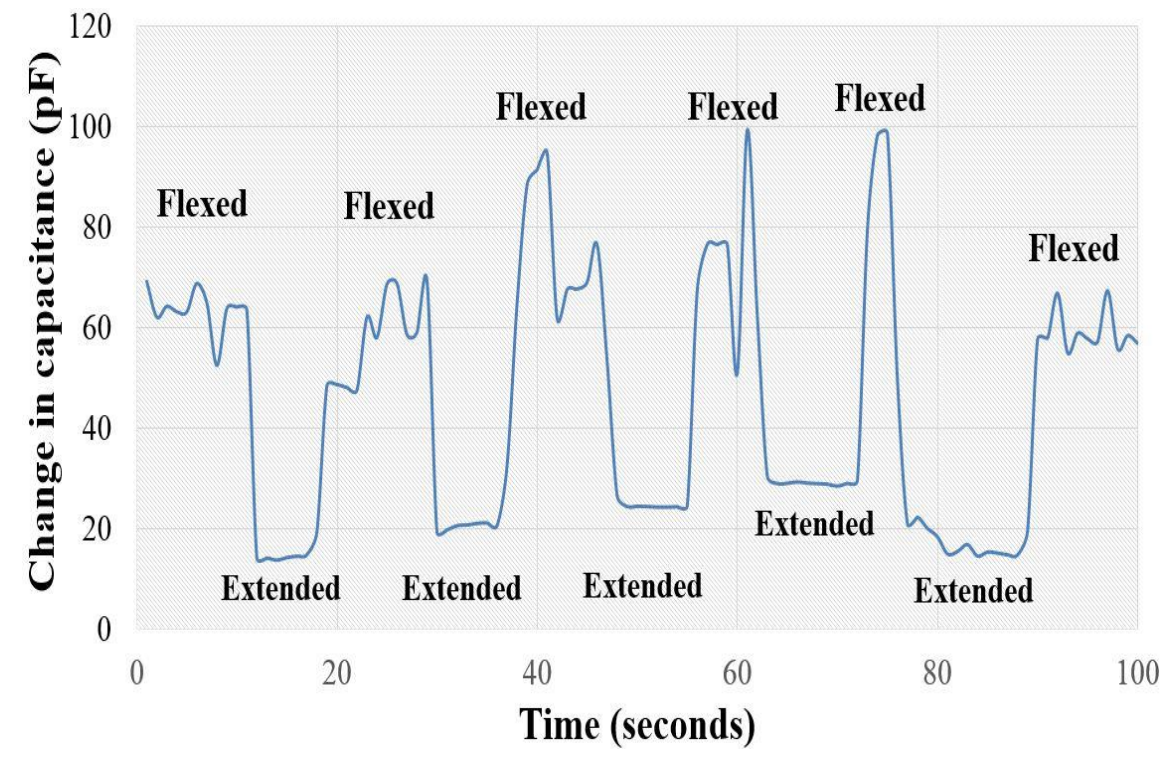

Figure 0.16 Detection of right-leg movement when the subject is in motion. 


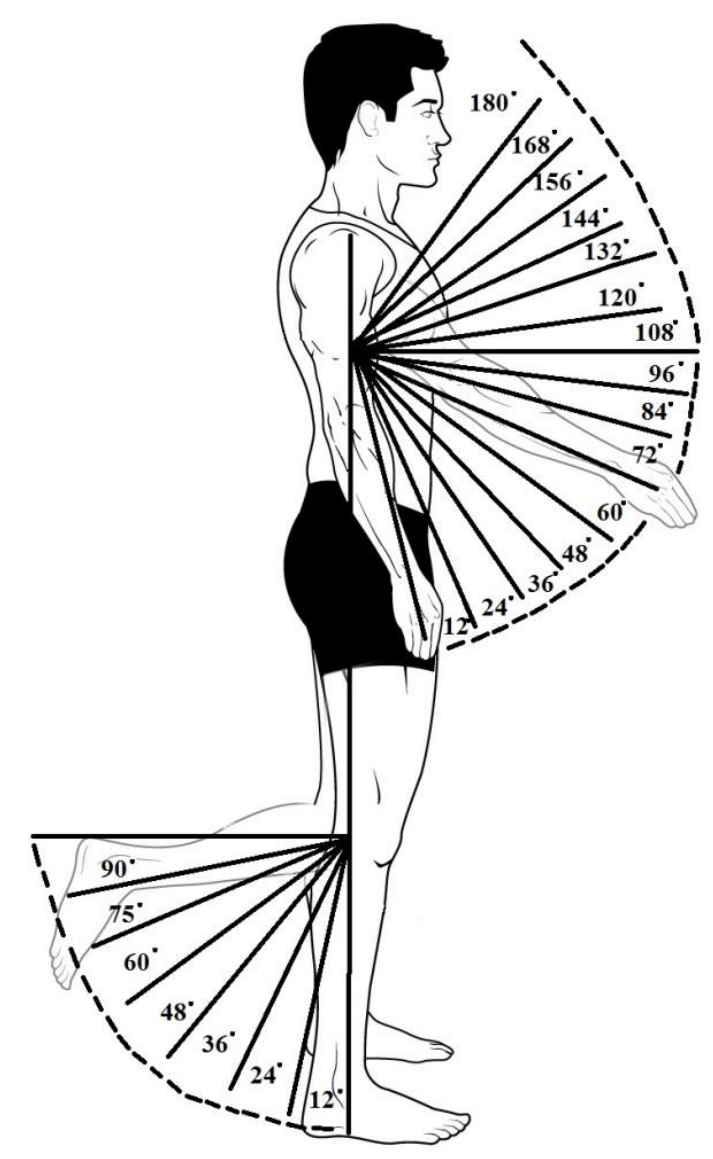

Figure 0.17 Different angular measurements of the limbs of a subject.

Not much difference is found for this case in comparison to the output when the subject is at rest. The differences between the two conditions for the flexed and extended situations can still easily be distinguished. The different changes in the angle concerning a reference state of a limb of a subject are shown in Figure 4.17. The evaluations were done using a Winkeltronic angle finder $(450 \mathrm{~mm})$. This experiment was performed to quantify a relationship between the changes in the capacitance values on the change in each degree of the limb. It is seen from Figure 4.18 that the changes of capacitance 
values are linear concerning the change in the degree of the limbs. The maximum angular variation concerning the reference was considered up to $130^{\circ}$ due to restricted bending of the limbs.

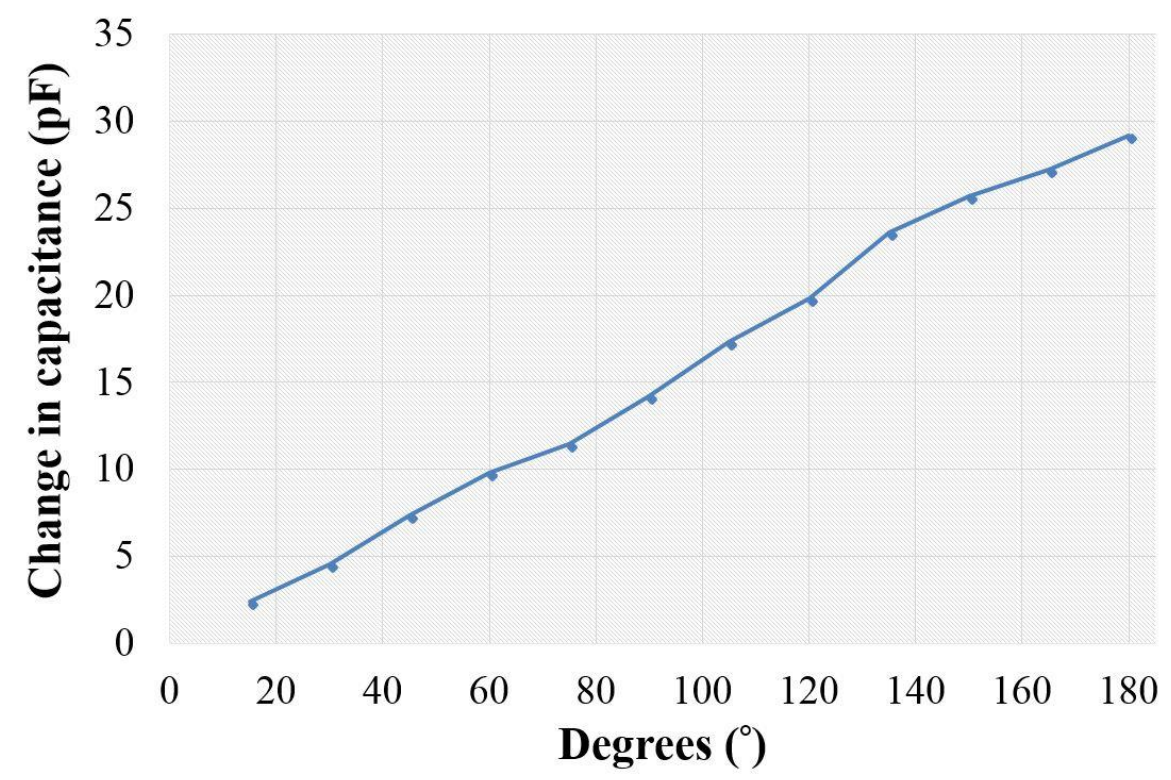

Figure 0.18 Change of capacitance as a function of the right-arm limb movement.

Figure 4.19 showed the sensor response when it was used for the detection of respiratory activity. The readings shown in two colors represent two different individuals. Figure 4.20 shows the three respiration signals taken at different rates. The contraction and expansion occurring on the sensor patch are due to the movement of the diaphragm, which results in the change in the interdigital distance $(d)$ and total area $(A)$ of the sensing surface of the sensor patch. Hence, the sensor patch can precisely distinguish the different rates of inhalation and exhalation lead by a prominent change in the capacitance values. The inhale and exhale rates were also varied in a controlled fashion to further determine the sensitivity of the sensor patch as depicted in Figure 4.21. 


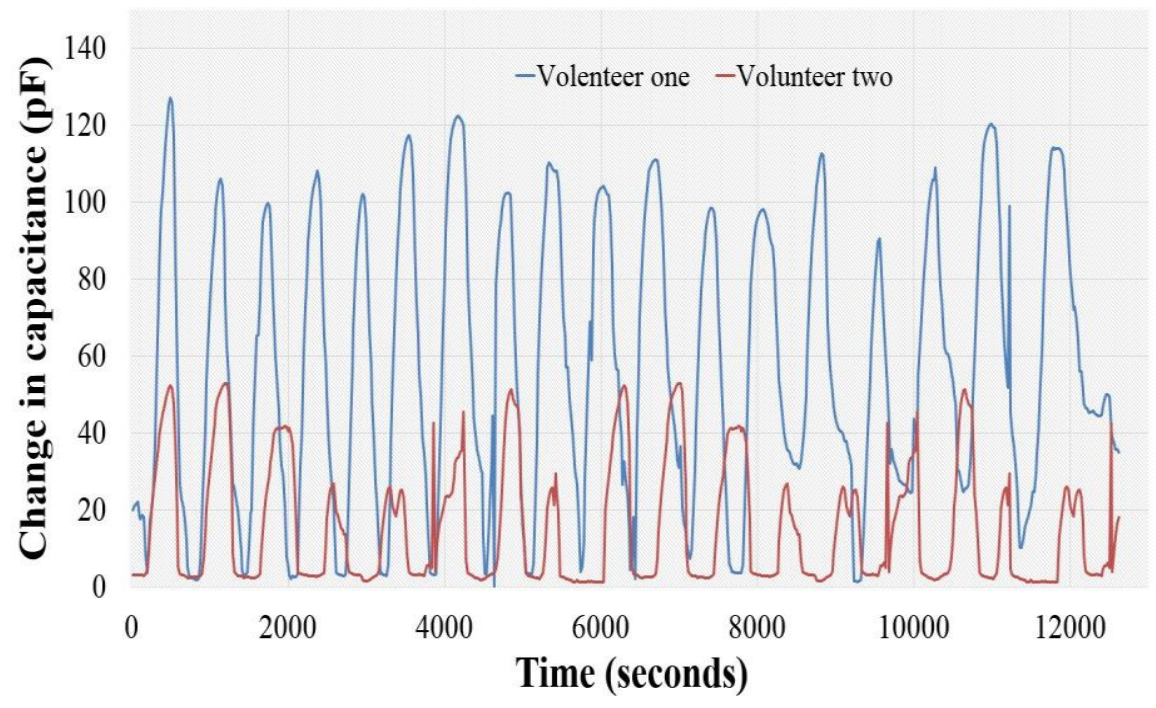

Figure 0.19 Monitoring of respiration for two different individuals at rest.

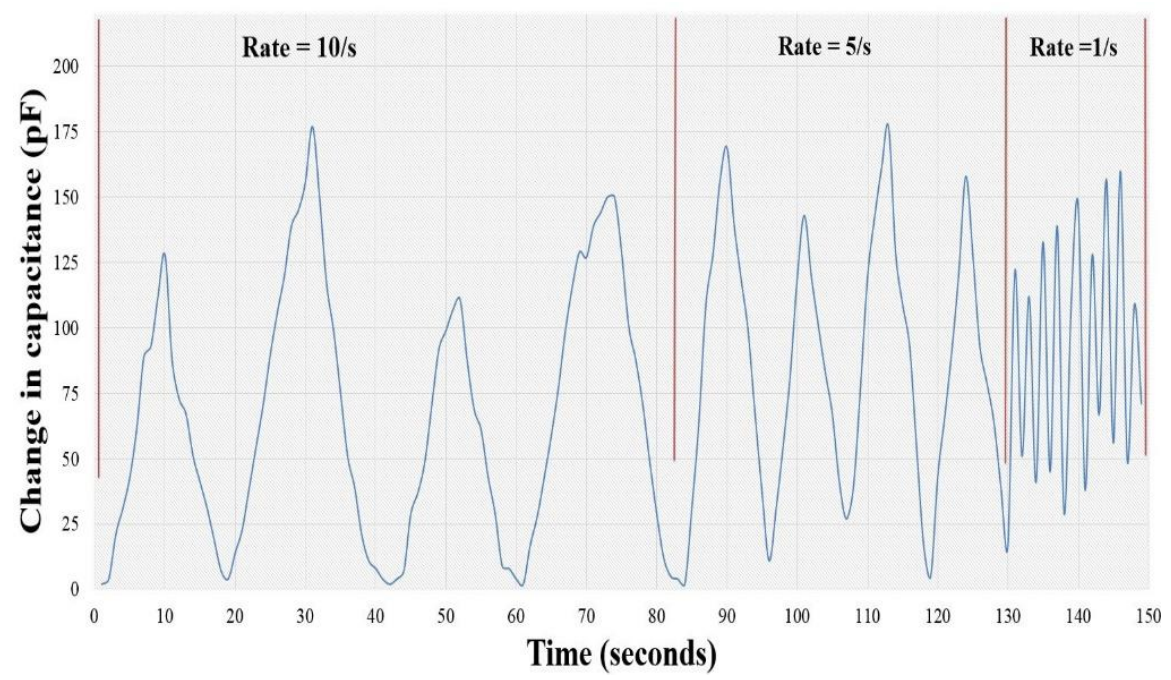

Figure 0.20 Measurement of respiration activity of an individual who was simulating respiration rates of $10 / \mathrm{s}$ for the first $80 \mathrm{~s}, 5 / \mathrm{s}$ for the next $45 \mathrm{~s}$ and $1 / \mathrm{s}$ for the next $20 \mathrm{~s}$. 


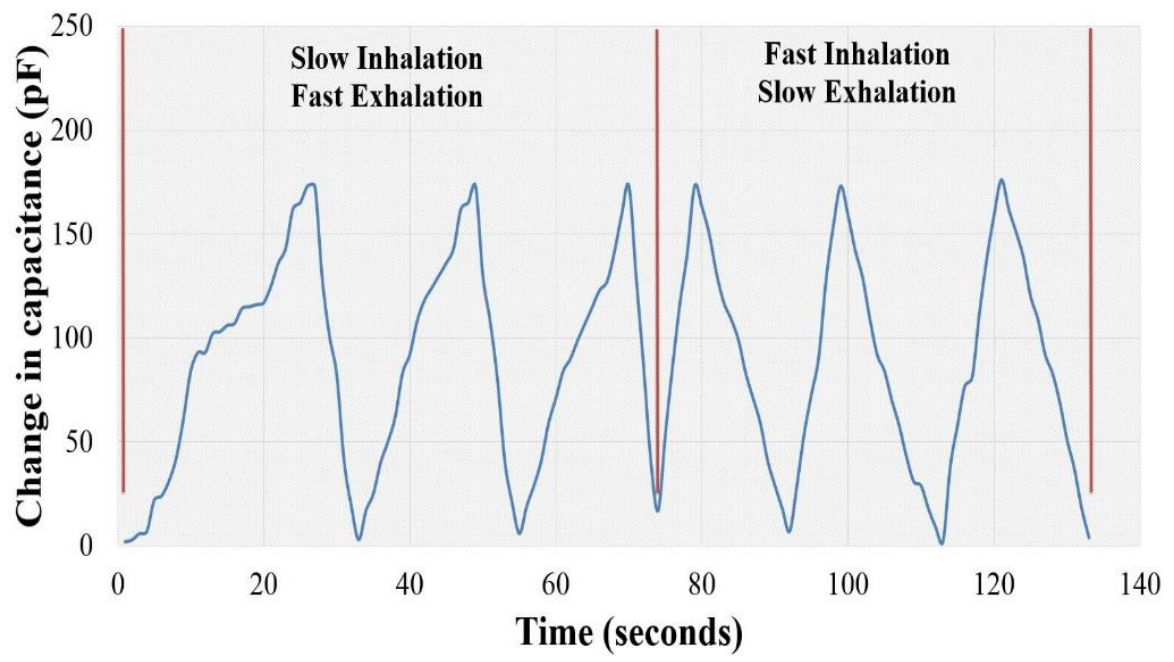

Figure 0.21 Measurement of respiration activity of an individual who was simulating inhalation and exhalation at different speeds.

\subsubsection{Conclusion}

Using carboxylic acid functionalized MWCNTs as a conductive material to develop the electrodes and PDMS as substrates, flexible sensors had been designed and fabricated. The MWCNTs were chosen as a filler to form the nanocomposite due to their advantages like high electrical conductivity, high mechanical flexibility, and high aspect ratio. The functionalization groups like $-\mathrm{COOH},-\mathrm{C}=\mathrm{O}$ and other oxygen carboxyl groups assist in increasing electrical conductivity and allows better dispersion of the nanotubes in the polymer matrix (Lau et al. 2008). PDMS has a low Young's modulus that has a high-performance patch for its robustness and durability. It is cheaper in comparison to other similar polymers like Polyethylene naphthalate (PAN), Polyethylene terephthalate (PET), that are commonly used commonly to develop flexible sensors. Due to its hydrophobicity, PDMS as a sensor substrate reduces the effects of sweat or other liquids on the response of the sensor output and its attachment to its skin. Another advantage of these sensor patches is their smaller sizes, which is around $50 \mathrm{~mm}^{2}$, thus making them convenient for monitoring physiological parameters of elderly people or infants while reducing discomfort to a large extent. 


\subsection{Tactile Sensing}

The prosthesis has been considered for a long time as a replacement for a missing part of an amputee's body with an artificially developed one. The most common category of prosthesis done in the medical field is the prosthesis of limbs (Erik Scheme MSc and Kevin Englehart PhD 2011; Fougner et al. 2012; Muzumdar 2004), where different amputee parts of the upper or lower portions of the body are placed or replaced to help in restoring a normal life to the affected person. The common materials used for developing the prosthetic organs are fiberglass, Nylon, Dacron, Carbon, and Kevlar (Fortino et al. 2013). Controlling the stiffness and strength of the resultant organs are some of the basic advantages of these materials. But certain disadvantages like their brittle nature and the inability to remold the device once formed deters their applications. For example, the use of Dacron in prosthetic devices can lead to the inability of the original amputated region to repair and grow due to the stiffness of the prosthetic organ. This adversely effects on the growth of tissue of the affected portion which can eventually lead to multiple replacements. In the case of Kevlar, it has been difficult to shape the prosthetic material into the form of the amputated organ unless it is being operated with special tools and equipment. This increases the cost of the overall treatment (Odame and $\mathrm{Du}$ ). Kevlar also corrodes when they are in contact with chlorine. Thus, it is state-of-the-art to develop sensors that can be considered as a replacement for the commercially used prosthetic devices.

Touch-sensitive prosthetic limbs are an interesting development in the prosthetic sector where substantial work (Boland 2010; Carrozza et al. 2003) has been done over the past decade. There is a constant effort from the researchers develops touch-sensitive somatosensory organs which would feedback the sensory signals to the amputee's brain. The devices that have been developed tested are so far on robots (Cannata et al. 2008; Dargahi 2000) and artificially fabricated organs (Trampuz et al. 2003; Zimmerli 2006). An attempt was made (Touch-Sensitive Prosthetic Limbs Take Step Forward in Monkey Study 2013) to fabricate a brain-machine interfaced prosthetic limb. The high cost of fabrication, complex testing systems, and weak sensor response are some of the demerits of such systems. 
In the succeeding sections, the use of the fabricated CNT-PDMS sensor patches for tactile sensing has been described.

\subsubsection{Experimental Setup}

The performances of the sensors were evaluated by deploying it as a tactile sensor via attaching it at the tip of the forefinger. The patch was connected to the LCR meter via Kelvin probes as shown in Figure 4.22 .

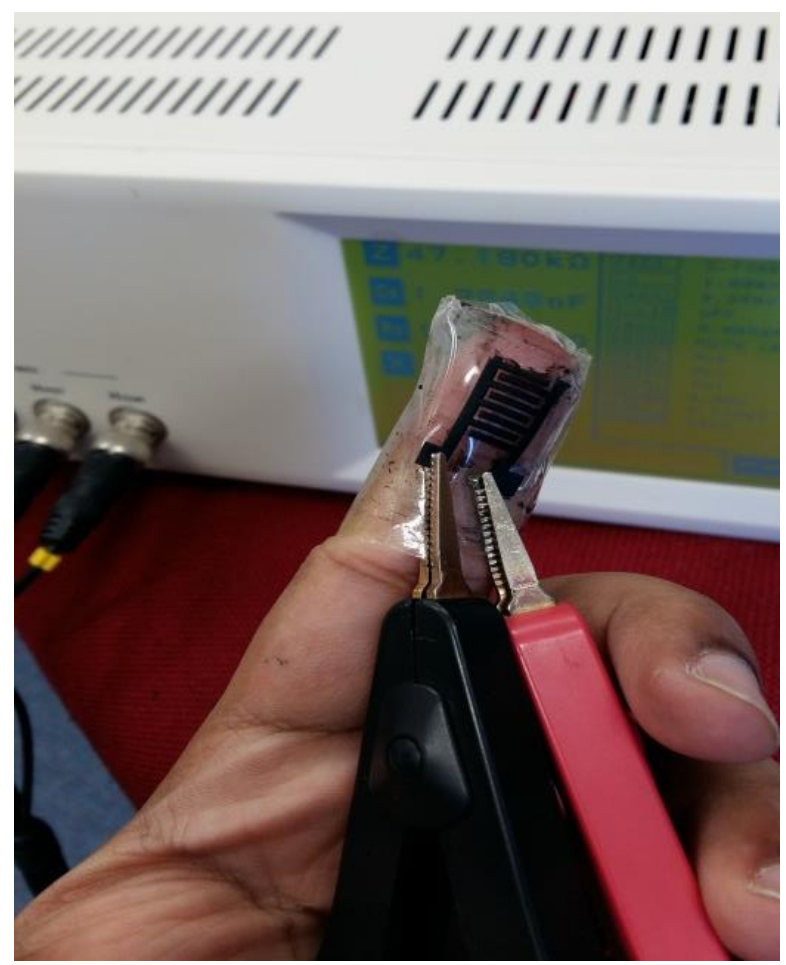

Figure 0.22 The sensor patch attached to the fingertip and connected to the LCR meter for tactile sensing.

An alternating voltage of $1 \mathrm{~V}$ peak-to-peak was provided as an input signal to the connected sensor patch. The change in response was determined to analyse the changes in capacitance in $\mathrm{pF}$ concerning time. 


\subsubsection{Results and Discussion}

Figures 4.23 - 4.27 show the sensor responses for two conditions; touch and no touch as shown by 'touched' and 'non-touched' conditions. The results are shown in the change of absolute capacitance values against time. Touched is considered for the situation when the sensor attached to the finger is pressed against a plane at different pressures. Not touched is considered for the situation when no stress is applied to the sensor. The results differed in the range of capacitance values during the touched condition as a result of the application of different pressures on the finger on the object. It is seen that the sensor patch responded well to the two defined conditions where the touched condition obtained higher values, thus distinguishable from the no-touch condition. The change in capacitance values with different weights is shown in Figure 4.28. Different weights were positioned on the sensing area of the patch to determine the changes in capacitance with different weights. It is seen from the Figure that the capacitance values change linearly concerning the change in weights.

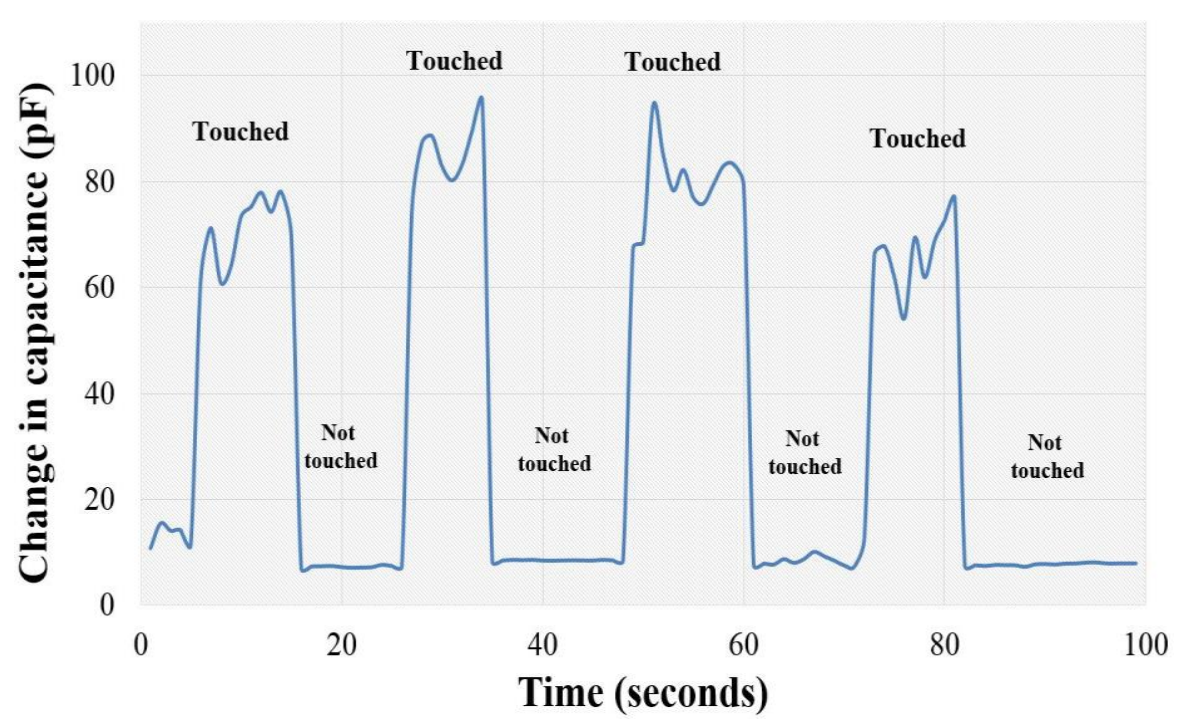

Figure 0.23 Response of the sensor patch for a pressure of $42.2 \mu \mathrm{Pa}$. 


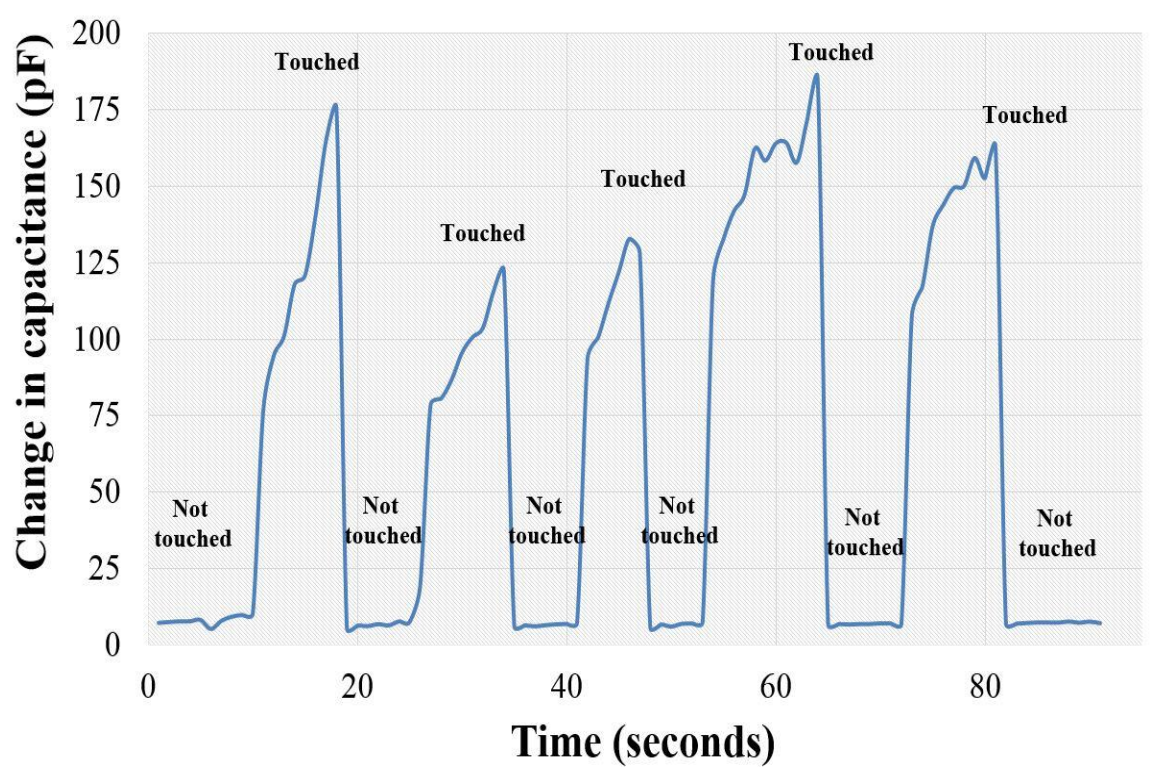

Figure 0.24 Response of the sensor patch for a pressure of $54.6 \mu \mathrm{Pa}$.

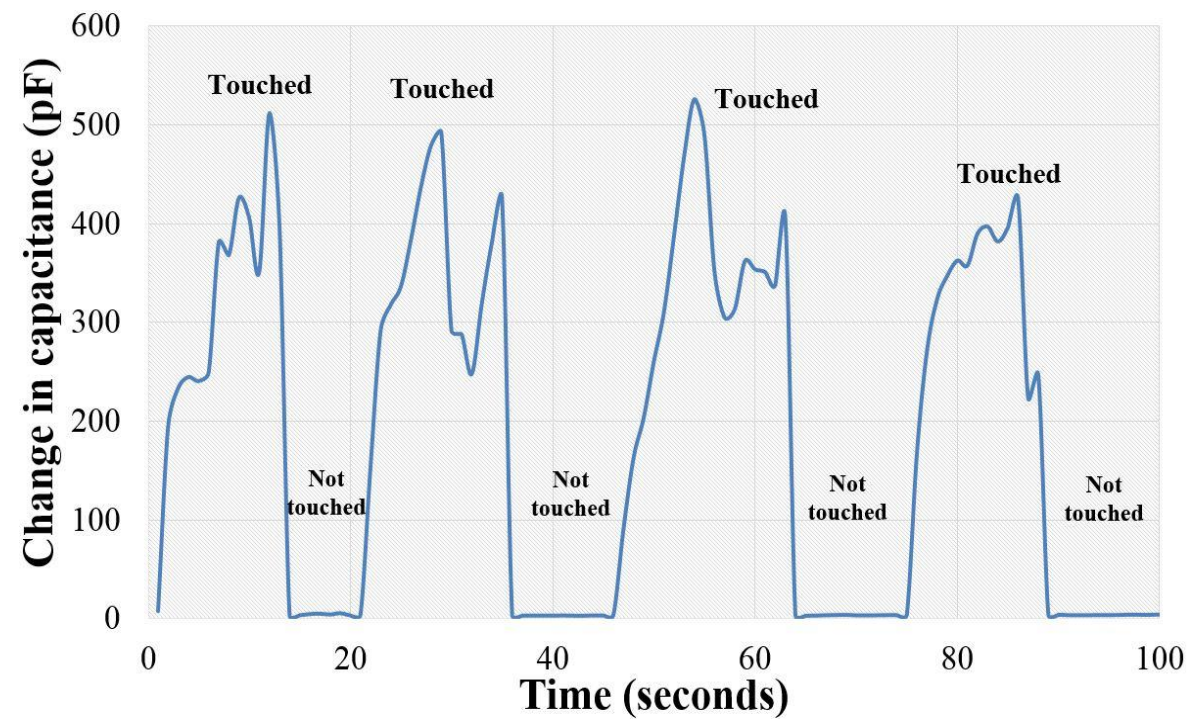

Figure 0.25 Response of the sensor patch for a pressure of $68.2 \mu \mathrm{Pa}$. 


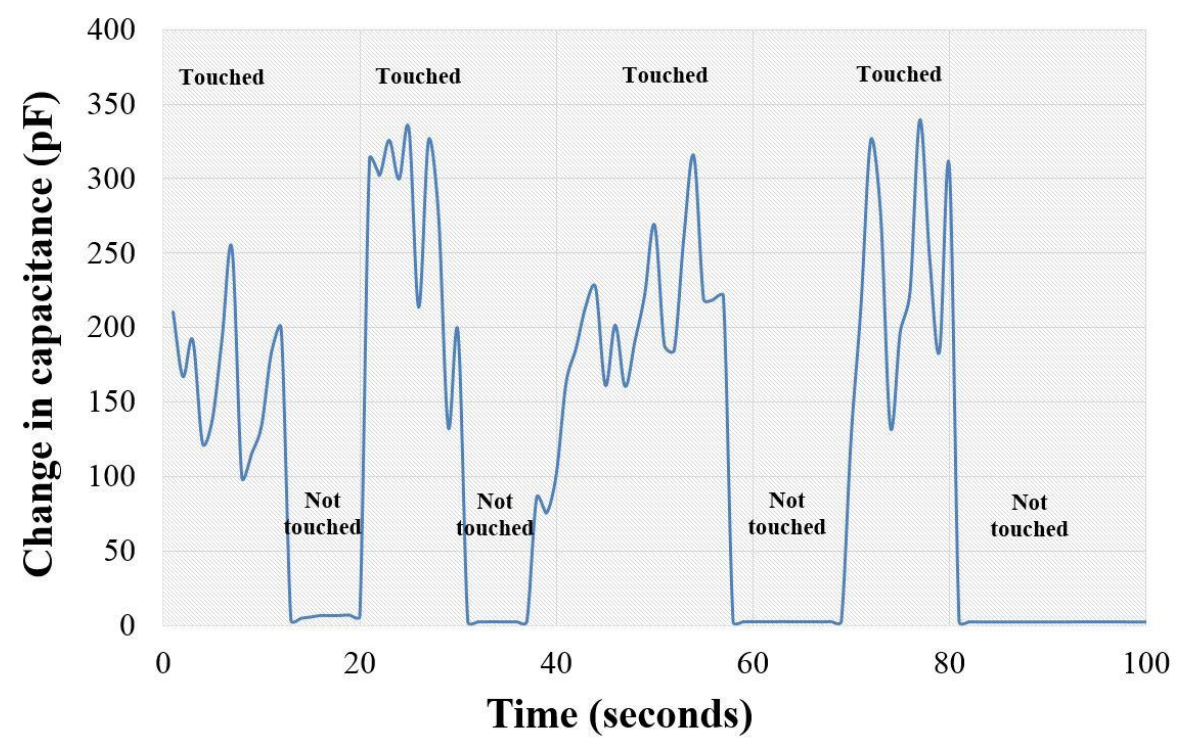

Figure 0.26 Response of the sensor patch for a pressure of $62.4 \mu \mathrm{Pa}$.

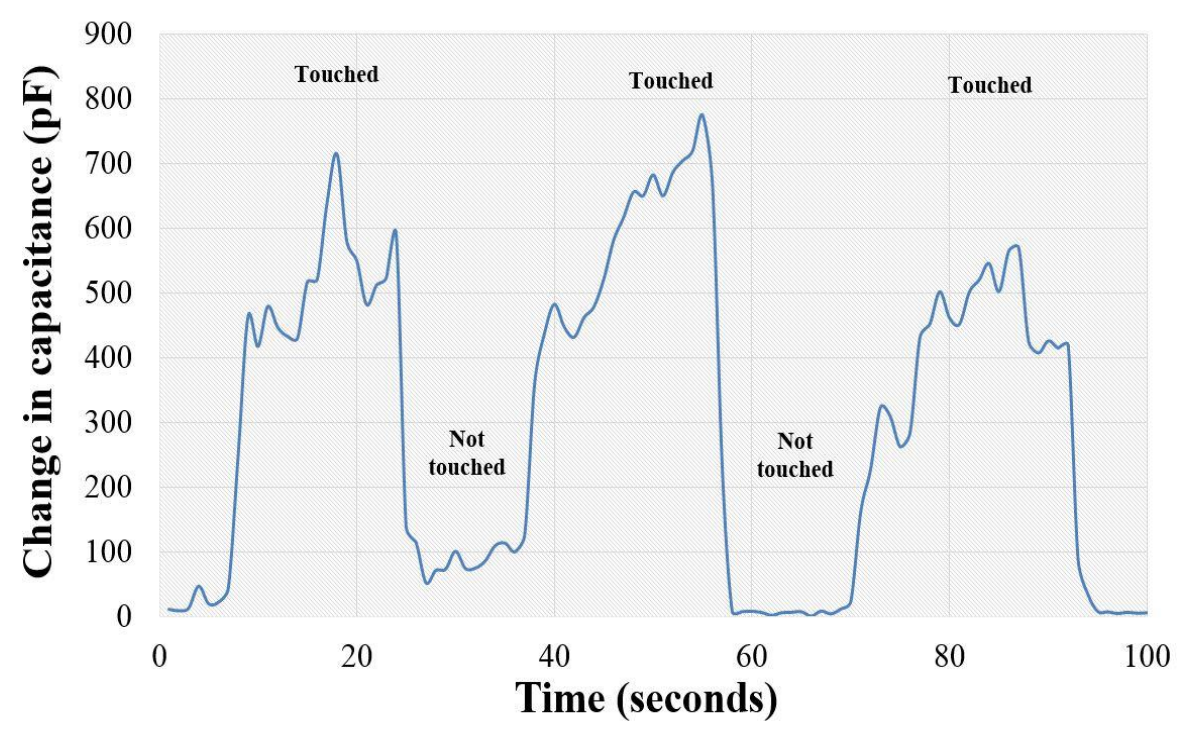

Figure 0.27 Response of the sensor patch for a pressure of $84.6 \mu \mathrm{Pa}$.

There were some glitches that were observed during the touched condition, which could be due to the aberrations related to the experimental setup, where the pressure applied on the finger was not constant. Also, since the connection of the sensor patches were done 
using tapes at the tip of the finger, these tapes would have loosened during the bending of the patch, which resulted in the sudden change in capacitance. The capacitance values in the no-touch condition have some non-zero values. This is due to the minimum strain caused on the sensor patch as a result of the bending curvature of the finger.

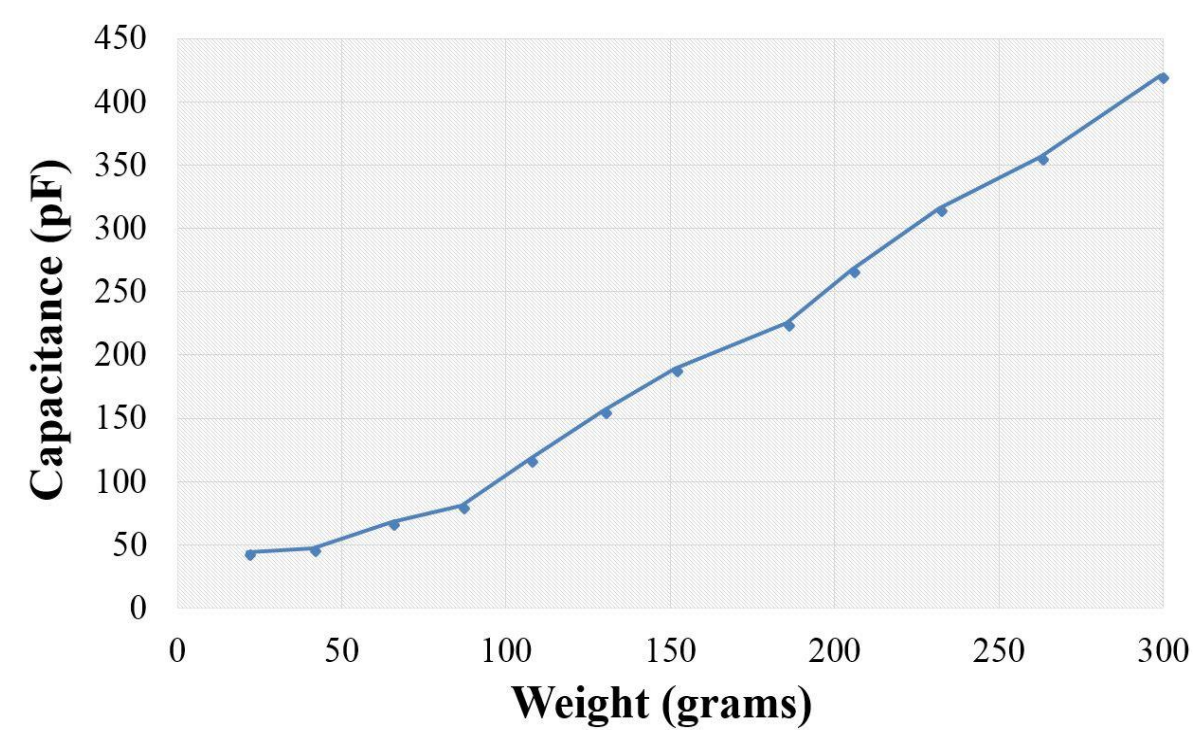

Figure 0.28 Variation of capacitance for different weight values.

\subsubsection{Conclusion}

The application of the CNT-PDMS sensor patches for tactile sensing has been described in the above section. Manual forces were exerted on the sensing area of the patches to analyze their respective responses towards low pressure. The experimental results obtained are very promising that could be based upon to extend the work behind this proposal. This idea could reduce the cost of fabrication cost and equip the existing prosthetic organs with efficient and high-sensitive tactile sensors. The next step would be to deploy these sensor prototypes in real-time applications to validate their functionality via embedding them to a signal-conditioning circuit to develop a complete prosthetic system for human uses. 


\subsection{Chapter Summary}

The design, fabrication of the CNT-PDMS sensor patches and their implementation of two different applications were described in this chapter. Due to the mechanical flexibility of the sensor patches, they were being employed for different types of applications like monitoring of physiological parameters and tactile sensing. Some of the major advantages of these sensor patches lie in their low cost of fabrication, simple operating principle, and multiple functionalities. The potentiality of these developed sensor patches regarding the applications can be extended for ubiquitous monitoring of elderlycare purposes to improve the quality of human life. Apart from limb movements and respiration, the detection of other physiological parameters like heart monitoring, fall detection, etc. can also be considered with these sensor patches. The embedding of these patches can be done to make them portable and point-of-care devices, which would be applicable to the ubiquitous monitoring of the daily activities of a person. The above-mentioned explanation of these sensor patches and their future opportunities does make them a viable option to be considered for using them in multifunctional sensing systems.

\subsection{References}

Altun K, Barshan B (2010) Human activity recognition using inertial/magnetic sensor units. In: Human behavior understanding. Springer, pp 38-51

Aminian K, Najafi B (2004) Capturing human motion using bodyfixed sensors: outdoor measurement and clinical applications Computer Animation and Virtual Worlds 15:79-94

Arena A, Donato N, Saitta G, Bonavita A, Rizzo G, Neri G (2010) Flexible ethanol sensors on glossy paper substrates operating at room temperature Sensors and Actuators B: Chemical $145: 488-494$

Armani D, Liu C, Aluru N Re-configurable fluid circuits by PDMS elastomer micromachining. In: Micro Electro Mechanical Systems, 1999. MEMS'99. Twelfth IEEE International Conference on, 1999. Ieee, pp 222-227 
Ashruf C (2002) Thin flexible pressure sensors Sensor Review 22:322-327

Bell J, Shen X, Sazonov E Early detection of sit-to-stand transitions in a lower limb orthosis. In: Engineering in Medicine and Biology Society (EMBC), 2015 37th Annual International Conference of the IEEE, 2015. IEEE, pp 5028-5031

Boland JJ (2010) Flexible electronics: Within touch of artificial skin Nature materials 9:790-792

Briand D, Molina-Lopez F, Quintero AV, Ataman C, Courbat J, de Rooij NF (2011) Why going towards plastic and flexible sensors? Procedia engineering 25:8-15

$\mathrm{Bu} \mathrm{N}$, Ueno N, Fukuda O Monitoring of respiration and heartbeat during sleep using a flexible piezoelectric film sensor and empirical mode decomposition. In: 2007 29th Annual International Conference of the IEEE Engineering in Medicine and Biology Society, 2007. IEEE, pp 1362-1366

Cannata G, Maggiali M, Metta G, Sandini G An embedded artificial skin for humanoid robots. In: Multisensor Fusion and Integration for Intelligent Systems, 2008. MFI 2008. IEEE International Conference on, 2008. IEEE, pp 434-438

Carrozza MC, Dario P, Vecchi F, Roccella S, Zecca M, Sebastiani E The CyberHand: on the design of a cybernetic prosthetic hand intended to be interfaced to the peripheral nervous system. In: Intelligent Robots and Systems, 2003.(IROS 2003). Proceedings. 2003 IEEE/RSJ International Conference on, 2003. IEEE, pp 2642-2647

Charton C, Schiller N, Fahland M, Holländer A, Wedel A, Noller K (2006) Development of high barrier films on flexible polymer substrates Thin Solid Films 502:99-103

Chiu Y-Y, Lin W-Y, Wang H-Y, Huang S-B, Wu M-H (2013) Development of a piezoelectric polyvinylidene fluoride (PVDF) polymer-based sensor patch for simultaneous heartbeat and respiration monitoring Sensors and Actuators A: Physical 189:328-334

Crabtree VM, Ivanenko A, O'Brien LM, Gozal D (2003) Periodic limb movement disorder of sleep in children Journal of Sleep Research 12:73-81 
Dargahi J (2000) A piezoelectric tactile sensor with three sensing elements for robotic, endoscopic and prosthetic applications Sensors and Actuators A: Physical 80:23-30

De Gans B-J, Duineveld PC, Schubert US (2004) Inkjet printing of polymers: state of the art and future developments Advanced materials 16:203-213

Erik Scheme MSc P, Kevin Englehart PhD P (2011) Electromyogram pattern recognition for control of powered upper-limb prostheses: State of the art and challenges for clinical use Journal of rehabilitation research and development 48:643

Fortino G, Giannantonio R, Gravina R, Kuryloski P, Jafari R (2013) Enabling effective programming and flexible management of efficient body sensor network applications Human-Machine Systems, IEEE Transactions on 43:115-133

Fougner A, Stavdahl Ø, Kyberd PJ, Losier YG, Parker PA (2012) Control of upper limb prostheses: terminology and proportional myoelectric control-a review Neural Systems and Rehabilitation Engineering, IEEE Transactions on 20:663-677

Frankland S, Harik V, Odegard G, Brenner D, Gates T (2003) The stress-strain behavior of polymer-nanotube composites from molecular dynamics simulation Composites Science and Technology 63:1655-1661

Fujii T (2002) PDMS-based microfluidic devices for biomedical applications Microelectronic Engineering 61:907-914

Gower MC Excimer laser microfabrication and micromachining. In: First International Symposium on Laser Precision Microfabrication (LPM2000), 2000. International Society for Optics and Photonics, pp 124-131

Hayes TL, Hagler S, Austin D, Kaye J, Pavel M Unobtrusive assessment of walking speed in the home using inexpensive PIR sensors. In: Engineering in Medicine and Biology Society, 2009. EMBC 2009. Annual International Conference of the IEEE, 2009. IEEE, pp 7248-7251

Herzer N, Hoeppener S, Schubert US (2010) Fabrication of patterned silane based self-assembled monolayers by photolithography and surface reactions on silicon-oxide substrates Chemical Communications 46:5634-5652 
Jo B-H, Van Lerberghe LM, Motsegood KM, Beebe DJ (2000) Threedimensional micro-channel fabrication in polydimethylsiloxane (PDMS) elastomer Microelectromechanical Systems, Journal of 9:76-81

Jung S, Ji T, Varadan VK (2006) Point-of-care temperature and respiration monitoring sensors for smart fabric applications Smart Materials and Structures 15:1872

Kaniusas E et al. (2006) Method for continuous nondisturbing monitoring of blood pressure by magnetoelastic skin curvature sensor and ECG Sensors Journal, IEEE 6:819-828

Kelly SDT, Suryadevara NK, Mukhopadhyay SC (2013) Towards the implementation of IoT for environmental condition monitoring in homes Sensors Journal, IEEE 13:3846-3853

Lam CXF, Mo X, Teoh S-H, Hutmacher D (2002) Scaffold development using 3D printing with a starch-based polymer Materials Science and Engineering: C 20:49-56

Lau CH et al. (2008) The effect of functionalization on structure and electrical conductivity of multi-walled carbon nanotubes Journal of Nanoparticle Research 10:77-88

Leonard PA, Douglas JG, Grubb NR, Clifton D, Addison PS, Watson JN (2006) A fully automated algorithm for the determination of respiratory rate from the photoplethysmogram Journal of Clinical Monitoring and Computing 20:33-36

Malhi K, Mukhopadhyay SC, Schnepper J, Haefke M, Ewald H (2012) A Zigbee-based wearable physiological parameters monitoring system Sensors Journal, IEEE 12:423-430

Mehnen L et al. (2004) Magnetostrictive bilayer sensors-a survey Journal of alloys and compounds 369:202-204

Merritt CR, Nagle HT, Grant E (2009) Textile-based capacitive sensors for respiration monitoring IEEE Sensors journal 9:7178

Muzumdar A (2004) Powered Upper Limb Prostheses: Control, Implementation and Clinical Application; 11 Tables. Springer Science \& Business Media,

Najafi B, Aminian K, Paraschiv-Ionescu A, Loew F, Büla CJ, Robert P (2003) Ambulatory system for human motion analysis using a kinematic sensor: monitoring of daily physical activity in the 
elderly Biomedical Engineering, IEEE Transactions on 50:711-723

Nguyen KD, Chen I, Luo Z, Yeo SH, Duh HB-L (2011) A wearable sensing system for tracking and monitoring of functional arm movement Mechatronics, IEEE/ASME Transactions on $16: 213-220$

Nilsson L, Johansson A, Kalman S (2000) Monitoring of respiratory rate in postoperative care using a new photoplethysmographic technique Journal of Clinical Monitoring and Computing 16:309-315

Odame K, Du D Towards a smart sensor interface for wearable cough monitoring

Pfützner H et al. (2006) Magnetostrictive bilayers for multi-functional sensor families Sensors and Actuators A: Physical 129:154158

Reinvuo T, Hannula M, Sorvoja H, Alasaarela E, Myllylä R Measurement of respiratory rate with high-resolution accelerometer and EMFit pressure sensor. In: Sensors Applications Symposium, 2006. Proceedings of the 2006 IEEE, 2006. IEEE, pp 192-195

Sazonov ES, Fulk G, Hill J, Schutz Y, Browning R (2011) Monitoring of posture allocations and activities by a shoe-based wearable sensor Biomedical Engineering, IEEE Transactions on 58:983-990

Snakenborg D, Klank H, Kutter JP (2004) Microstructure fabrication with a $\mathrm{CO} 2$ laser system Journal of Micromechanics and microengineering 14:182

Suryadevara NK, Mukhopadhyay SC (2012) Wireless sensor network based home monitoring system for wellness determination of elderly Sensors Journal, IEEE 12:1965-1972

Touch-Sensitive Prosthetic Limbs Take Step Forward in Monkey Study. (2013). http://www.livescience.com/40405-touchsensitive-prosthetic-limbs-monkey-study.html.

Trampuz A, Steckelberg JM, Osmon DR, Cockerill Iii FR, Hanssen AD, Patel R (2003) Advances in the laboratory diagnosis of prosthetic joint infection Reviews in Medical Microbiology $14: 1-14$ 
Warkentin M, Freese HM, Karsten U, Schumann R (2007) New and fast method to quantify respiration rates of bacterial and plankton communities in freshwater ecosystems by using optical oxygen sensor spots Applied and environmental microbiology 73:6722-6729

Yang C-C, Hsu Y-L (2010) A review of accelerometry-based wearable motion detectors for physical activity monitoring Sensors 10:7772-7788

Zimmerli W (2006) Prosthetic-joint-associated infections Best Practice \& Research Clinical Rheumatology 20:1045-1063 